\title{
Evaluación del riesgo de inundación ante tsunami local en la isla de Cascajal, Pacífico Colombiano'
}

\author{
Danny Alexander Cocuñame Ricardo² y Elkin de Jesús Salcedo Hurtado³
}

\begin{abstract}
RESUMEN
La Isla de Cascajal es un importante lugar geográfico en el Pacífico colombiano, donde se emplazan las instalaciones e infraestructura administrativa del sistema gubernamental y portuario del municipio de Buenaventura. Se encuentra bajo amenaza por inundación ante un tsunami local por la posible ocurrencia de un fuerte terremoto. Considerando la amenaza sísmica regional se determinan escenarios de inundación que evalúan la posible altura y el área cubierta por el nivel de las aguas en la Isla de Cascajal. El escenario más crítico ocurre ante a un evento tsunamigénico de magnitud $8,8 \mathrm{Mw}$, generando una inundación con altura de 2,88 metros, que cubriría $3,32 \mathrm{~km}^{2}$, equivalente al 94,6 " $\%$ del área total de la Isla. Este resultado se correlaciona directamente con los datos obtenidos respecto a la densidad poblacional y el tipo de actividades económicas como elementos expuestos susceptibles a verse afectados por el escenario de amenaza mencionado. Este análisis permite establecer el riesgo por inundación tsunamigénica para el centro poblado de la Isla de Cascajal, el cual puede tomarse como insumo para la planificación regional y la toma de medidas de prevención y mitigación en la zona de estudio.
\end{abstract}

Palabras clave: Amenaza sísmica, inundación, tsunami, vulnerabilidad, riesgo.

\begin{abstract}
Cascajal's Island is an important geographic place of the Colombian Pacific, where administrative facilities and infrastructure of the government and port system from the municipality of Buenaventura are established. It is consider under flooding hazard due to a local tsunami from possible occurrence of a strong earthquake. Considering the regional seismic hazard, flooding scenarios are determinate to evaluate the possible height and the covered area by water at Cascajal's Island. The most critical scenario is generate by a tsunamigenic event with magnitude $8,8 \mathrm{Mw}$, generating a flooding with a height of 2,88 meters that will cover $3,32 \mathrm{~km}^{2}$ equivalents to $94,6 \%$ of the Island's total area. This result is correlated directly with the acquired data about population density and kind of economic activities as exposure elements that are susceptibles to be affected by hazard scenario. This analysis let to establish risk by the tsunamigenic flood to the populated center of Cascajal, which can be taken as an input to regional planning and as prevention and mitigation measurements on the study zone.
\end{abstract}

Key words: Seismic hazard, flood, tsunamis, vulnerability, risk.

Los autores agradecen la colaboración de Gustavo Barrantes de la Universidad Nacional de Costa Rica, Emmanuel Zapata Caldas, miembro del grupo de investigación Territorios, y del profesor Andrés Quintero. También agradecen a la Dirección General Marítima, emplazada en la Capitanía del Puerto de Buenaventura, específicamente a la funcionaria Georgina García. Al personal del Observatorio Sismológico y Geofísico del Suroccidente Colombiano y del grupo de investigación GeoRiesgos. Este trabajo fue realizado dentro del proyecto "Caracterización del régimen sísmico y procesos de deformación cortical actual en el Valle del Cauca y zonas aledañas" financiado y registrado por la Vicerrectoría de Investigaciones de la Universidad del Valle con el código Cl 297. Artículo recibido el 23 de agosto de 2016, aceptado el 5 de diciembre de 2016 y corregido el 5 de marzo de 2017

Grupo Georiesgos del Observatorio Sismológico y Geofísico del Suroccidente Colombiano / Universidad del Valle (Colombia).

E-mail: danny.cocuname@correounivalle.edu.co

Departamento de Geografía, Universidad del Valle (Colombia).E-mail: elkin.salcedo@correounivalle.edu.co 
La Isla de Cascajal, localizada en el Pacífico colombiano, corresponde al sector urbano del municipio de Buenaventura, donde se emplazan las instalaciones e infraestructura administrativa del sistema gubernamental y portuario existente para el municipio, y donde además se localiza el principal puerto marítimo del país en la región.

El Pacífico colombiano, se caracteriza por ser la zona de convergencia de las placas de Nazca y Sudamérica que genera fuertes terremotos capaces de producir tsunamis, que representan una grave amenaza para las comunidades asentadas en las márgenes o líneas de costa oceánica. En los últimos 120 años, en esta zona, se han presentado varios eventos sísmicos de magnitud considerable, algunos de ellos con potencial tsunamigénico como los de 1906, 1942, 1958 y 1979 (Figura № 1), siendo los más catastróficos los de 1906 y 1979 (Rudolph \& Szirtes, 1911; Kelleher, 1972; Kanamori, 1977; Ramírez y Goberna, 1980; Kanamori \& McNally, 1982).

Figura $\mathrm{N}^{\circ} 1$

Principales sismos ocurridos en la zona de subducción colombo - ecuatoriana

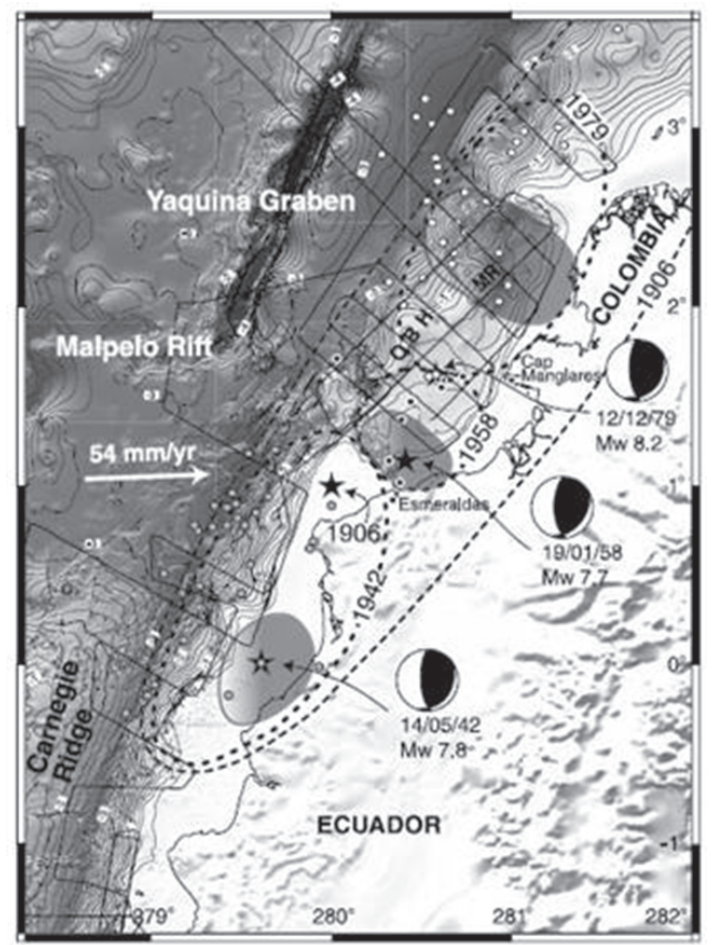

Fuente: Collot et al., 2004.

El primero, ocurrió el 31 de enero de 1906, con magnitud 8,8 Mw, la llegada de las olas causó la muerte de aproximadamente 1500 personas en las costas del departamento de Nariño, aunque las localidades más afectadas fueron Río Verde en Ecuador, junto a los municipios de Tumaco y López de Micay en Colombia (Rudolph \& Szirtes, 1911). El segundo evento se registró el 12 de diciembre de 1979, con magnitud 8,1 Mw, fue el terremoto más grande en el noroeste de Sudamérica desde 1942, y cuya ocurrencia podría explicarse para llenar una brecha sísmica en torno a 
la energía acumulada de sus antecesores, terremotos de 1906, 1942 y 1958 (Kelleher, 1972; Kanamori, 1977; Kanamori \& McNally, 1982). Este terremoto se sintió en ciudades localizadas al interior de Colombia como Bogotá, Cali, Popayán y Pasto, junto a Esmeraldas, Guayaquil y Quito del lado ecuatoriano (Herd et al., 1981). El tsunami generado por este terremoto, barrió literalmente casi todas las aldeas localizadas en la costa pacífica del suroccidente colombiano, afectando principalmente las costas de los departamentos de Cauca y Nariño, específicamente la zona comprendida entre Guapi y San Juan de la Costa, aunque otros sitios como El Charco y Mosquera también presentaron alto grado de destrucción (Pararas - Carayannis, 1980). Estos eventos permiten comprender el panorama de riesgo de inundación por tsunami para un foco económico y regional como el municipio de Buenaventura en el Valle del Cauca.

En Colombia, el modelamiento y análisis de eventos de tsunamis ha sido abordado por la Dirección General Marítima (2013), Caballero y Ortiz (2002), Restrepo y Otero (2007), cuyos estudios establecen zonas de inundación a partir de eventos tsunamigénicos para diversos lugares de la costa del suroccidente colombiano, incluida la Isla de Cascajal. Consideran escenarios de marea baja, media y alta, teniendo en cuenta como zona tsunamigénica el hipocentro del evento sísmico máximo registrado, sin el debido análisis de la amenaza sísmica en toda la zona del pacífico.

El presente trabajo establece la estimación del riesgo de inundación por tsunamis local en la isla de Cascajal, a partir de la generación de eventos sísmicos tsunamigénicos de orden probabilístico, los cuales se establecen por medio de un análisis de amenaza sísmica para las fuentes de subducción en el Pacífico colombiano. Lo que representa una contribución en el análisis de este tipo de fenómenos, ya que los estudios previos, no consideran el análisis de la amenaza sísmica para su desarrollo. El modelamiento se hace usando el programa CRISIS 2007 (Ordaz et al., 2007). Estudios similares para otras regiones del mundo han sido realizados por Papoulia et al. (2010) y (ERNA - Latina, 2011), cuyos resultados coinciden en la efectividad lograda al generar escenarios de inundación por tsunami a través de un análisis de amenaza sísmica.

Para este estudio se usó un modelo sismotectónico que divide la zona de subducción en tres subfuentes, donde cada una ellas actúa independientemente en la generación de un evento sísmico tsunamigénico. El modelamiento del nivel de inundación se establece a partir del evento tsunamigénico máximo que en su conjunto podrían generar las tres subfuentes sismogénicas.

\section{Eventos tsunamigénicos}

Un tsunami es entendido como el desplazamiento de cuerpos de agua asociados a una liberación inicial de energía por procesos de deformación. El desplazamiento de los cuerpos de agua se produce en sentido radial al punto de origen. Los procesos que generan la aparición de tsunamis son conocidos como eventos tsunamigénicos, tales como, las erupciones volcánicas (Choi et al., 2003), eventos de remoción en masa (Geist, 1998; Wieczorek et al., 2004, Siebert, 2004; Dengler et al., 2011), impactos cósmicos (Bryant, 2014; Poag et al., 2004; Warme \& Kuehner, 1998) y por terremotos tectónicos (Kanamori, 1972; Abe, 1989; Zitellini et al., 1999; Cardona et al., 2007; Tinti et al., 2011; Martínez et al., 2012; Martínez y Aránguiz, 2016). La evaluación realizada en este estudio se hace en base a este último tipo de eventos, es decir, los terremotos de origen tectónico. 
El proceso de generación y propagación de un tsunami generalmente es el resultado de un intercambio entre energía gravitacional y energía cinética horizontal en una columna de agua (Okal, 1988; Geist, 1999). En el Cuadro No 1, se especifican las características de generación, propagación e impacto, además del riesgo que se generaría para el proceso de ocurrencia de un tsunami de origen tectónico.

\section{Cuadro $\mathrm{N}^{\circ} 1$}

Procesos para la ocurrencia de eventos sísmicos tsunamigénicos

\begin{tabular}{|c|c|c|c|c|}
\hline \multirow{3}{*}{ 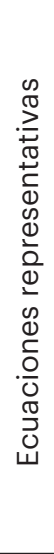 } & Generación & Propagación & Impacto (Run - Up) & Riesgo \\
\hline & $u_{m}=\iint D_{i} V_{j} U_{m}^{i j} d \Sigma$ & $\begin{array}{l}\frac{\partial(n+h)}{\partial t}+\nabla *[V(n+h)=0 \\
\frac{\partial V}{\partial t}+(V * \nabla) V+g \nabla n=0\end{array}$ & $\zeta(y)=\frac{b}{\left(\frac{y}{a}\right)^{2}+1}$ & $\begin{array}{l}\quad R T=A * V \\
\quad V=(E E) * F \\
\text { V - Vulnerabilidad } \\
\text { (Incluye VH, VS) } \\
\text { EE - Elementos } \\
\text { expuestos (Incluye } \\
\text { EH, ES). } \\
\text { F- Fragilidad }\end{array}$ \\
\hline & $\begin{array}{c}\text { Desplazamiento } \\
\text { para una dislocación } \\
\text { Somigliana arbitraria } \\
\text { (Rybicki, 1986) }\end{array}$ & $\begin{array}{c}\text { Ecuaciones de ondas } \\
\text { superficiales (Goto et al., } \\
\text { 1997) }\end{array}$ & $\begin{array}{l}\text { Modelamiento } \\
\text { de inundación } \\
\text { paramétrico (Okal } \\
\text { and Sinolakis, } \\
\text { 2004) } \\
\end{array}$ & $\begin{array}{c}\text { Definición general } \\
\text { de riesgo (Fournier } \\
\text { d'Albe, 1979, Grezio } \\
\text { et al., 2012) }\end{array}$ \\
\hline $\begin{array}{l}0 \\
\frac{0}{0} \\
\frac{\pi}{0} \\
0 \\
\frac{1}{0} \\
0 \\
0 \\
0 \\
0 \\
0 \\
0\end{array}$ & $\begin{array}{l}D_{i(x)}-\text { Distribución de } \\
\text { deslizamiento. } \\
\Sigma-\text { Área de ruptura. } \\
C_{i j d}-\text { Módulos de } \\
\text { ruptura. } \\
V_{f}-\text { Superficie } \\
\text { normal. }\end{array}$ & $\begin{array}{l}n \text { - Desplazamiento } \\
\text { vertical de la superficie } \\
\text { del agua. } \\
h \text { - Profundidad media. } \\
\text { g- Atracción } \\
\text { gravitacional. } \\
V \text { - Componentes } \\
\text { promedio de la } \\
\text { profundidad para las } \\
\text { velocidades horizontales. }\end{array}$ & $\begin{array}{l}y \text { - Distancia a la } \\
\text { costa. } \\
\varsigma(y)-\text { Altura } \\
\text { a - Distribución } \\
\text { de las olas sobre } \\
\text { el terreno de } \\
\text { afectación. } \\
\text { b-Altura máxima } \\
\text { de las olas } \\
\text { registradas. }\end{array}$ & $\begin{array}{l}R T-\text { Riesgo } \\
\text { tsunami. } \\
\text { A - Amenaza } \\
\text { tsunami } \\
\text { EH - Exp. Humano. } \\
\text { ES - Exp. } \\
\text { Estructural. } \\
\text { VH - Vul. Humana. } \\
\text { VS - Vul. } \\
\text { Estructural. }\end{array}$ \\
\hline
\end{tabular}

Fuente: Modificado de Geist (1999).

\section{Área de estudio}

El Distrito Especial, Industrial, Portuario, Biodiverso y Ecoturístico de Buenaventura, es un municipio colombiano ubicado en el departamento del Valle del Cauca. Es el principal puerto del Pacífico colombiano. Fue fundado por Juan Ladrillero el 14 de Julio de 1540, cuenta con una población de 327.955 habitantes, según datos proyectados al 2010, del Censo General de la Población Nacional (DANE, 2005a).

El área urbana y rural comprende aproximadamente el 30\% del Valle del Cauca, teniendo como límite la línea de costa en el océano Pacífico, hasta las altas cumbres de la cordillera Occi- 
dental de los Andes colombianos. Su casco urbano corresponde a la Isla de Cascajal (Buenaventura Insular), la cual incorpora dentro de su área las instalaciones e infraestructura administrativa del sistema gubernamental y portuario existente para el municipio de Buenaventura (Figura $N^{\circ} 2$ ).

Figura $\mathrm{N}^{\circ} 2$

Localización del área de estudio.

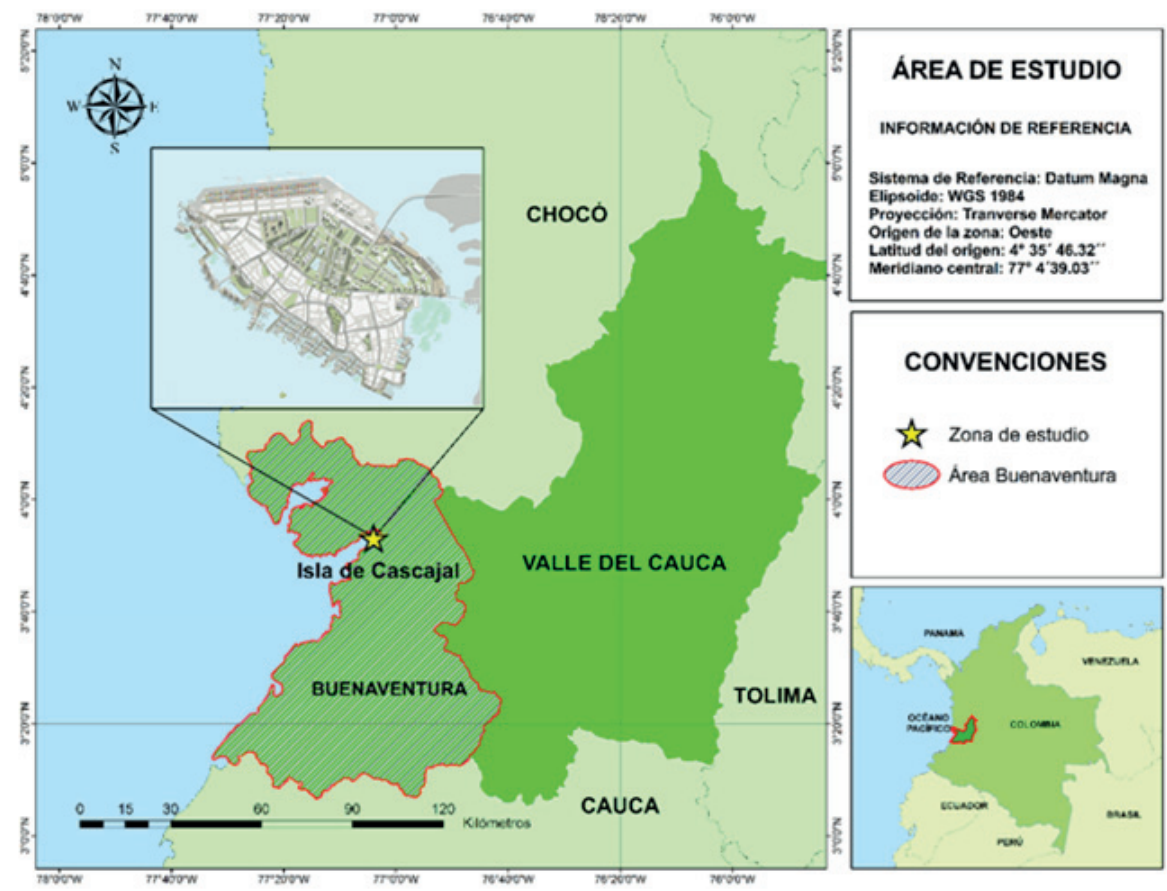

Fuente: Elaboración propia.

\section{Metodología}

Para el desarrollo de este trabajo, se realizó el cálculo probabilístico de la amenaza sísmica para el municipio de Buenaventura utilizando un modelo de peligro sísmico que incluye los diferentes parámetros que determinan la tasa de excedencia de la aceleración en el suelo a partir de la tasa de excedencia de las magnitudes registradas y su relación con los valores de atenuación preestablecidos para las fuentes sismogénicas (Aguilar, 2001).

La amenaza o peligro sísmico se comprende como la caracterización numérica de la probabilidad de ocurrencia de eventos sísmicos por medio de valores de aceleración del suelo en un área determinada durante un periodo de tiempo definido. Para el cálculo de la amenaza sísmica es necesario establecer: las fuentes sismogénicas próximas al sitio de estudio, los valores de recurrencia sísmica y los datos de atenuación para los movimientos en el terreno. El modelo de peligro sísmico establecido considera las tasas de excedencia por magnitud registrada y las distancias epicentrales en las fuentes sísmicas establecidas (Aguilar, 2001). 
El programa CRISIS 2007 permite establecer las condiciones de amenaza sísmica en una zona determinada, a partir de la identificación de las diferentes fuentes sísmicas, el registro sísmico y otros parámetros como la atenuación sísmica, las ordenadas espectrales y los valores para los diferentes periodos de retorno útiles en el análisis.

De igual manera, se determina un modelo de inundación por tsunami de orden paramétrico a partir de eventos sísmicos tsunamigénicos de amenaza crítica, que simplifica el proceso de modelación a tiempos de cálculo razonable a partir de las observaciones de Run - Up para fenómenos de inundación producidos por eventos tsunamigénicos relevantes (Okal \& Sinolakis, 2004).

El programa CRISIS 2007 requiere dos modelos de elevación digital que corresponden a la batimetría de la cuenca oceánica del Pacífico colombiano circunscrita en las fuentes sísmicas establecidas, y a la topografía de las áreas de estudio en la Isla de Cascajal, Buenaventura. Dichos modelos permiten establecer los escenarios de amenaza de inundación por tsunami, a partir del cálculo de la amenaza sísmica en Buenaventura y los eventos sísmicos de amenaza crítica establecidos para cada fuente en dicho cálculo.

Figura $\mathrm{N}^{\circ} 3$

Esquema del diseño metodológico

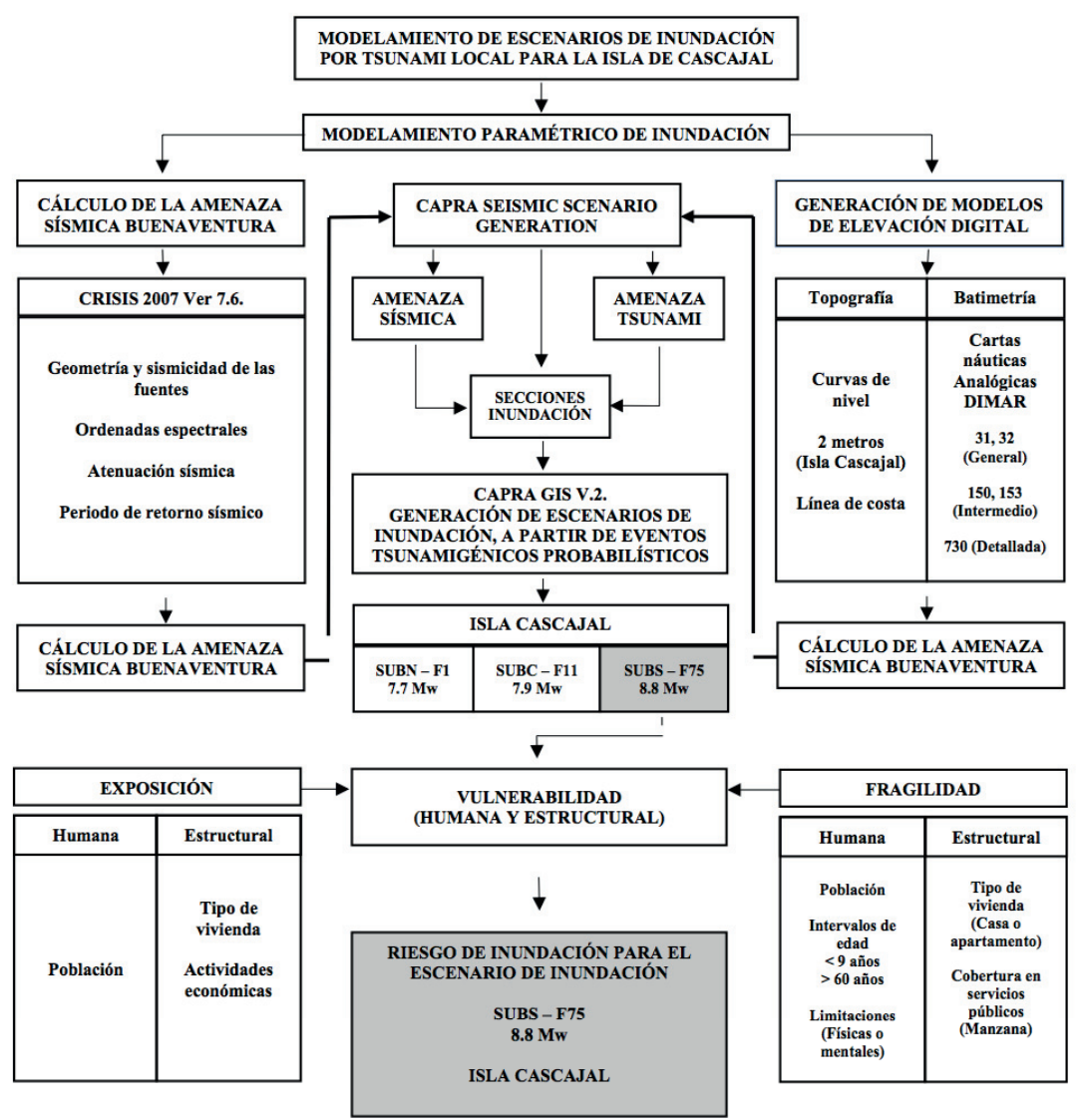

Fuente: Modificado de Cocuñame (2016). 
En la Figura $\mathrm{N}^{\circ} 3$, se presenta el esquema metodológico usado en este trabajo. El proceso se divide en dos pasos primordiales para el establecimiento de escenarios de inundación por tsunami local por medio del programa CRISIS 2007.

Determinado el escenario de amenaza, se establecen los elementos expuestos (Humanos y estructurales), y el grado de fragilidad para cada uno de ellos, con respecto al alcance máximo de la inundación. Finalmente, se evalúa el porcentaje de afectación acorde con el área inundable.

\section{Datos utilizados}

La información en formato digital requerida para el establecimiento de los escenarios de inundación por tsunami es la siguiente:

- Catálogo de eventos sísmicos asociados a las fuentes de subducción en Colombia, cuyas magnitudes y profundidades deriven en tsunamis locales en el pacífico. Para ellos se escogieron sismos de la base de datos del RSNC y NEIC, ubicados en la zona de subducción, con magnitud $\mathrm{Mw}>6.0$ y profundidad menor que 60 kilómetros.

- Modelo de fuentes sismotectónicas, donde los eventos tsunamigénicos son posibles. Para el caso del pacífico colombiano se ha seleccionado la zona de subducción o convergencia entre las placas de Nazca y Sudamérica. Esta a su vez es subdividida en tres subfuentes (INGEOMINAS - DAGMA, 2005). Para cada subfuente es necesario determinar las coordenadas geográficas de cada uno de sus vértices y los respectivos valores de profundidad en kilómetros. Para el área de estudio, se entiende que solamente los mecanismos de subducción permiten la ocurrencia de fenómenos telúricos de carácter tsunamigénico. Por lo tanto, los sistemas de fallamiento cortical, son completamente ignorados para el cálculo de la amenaza sísmica.

- Datos de información topográfica a escala detallada (1:8.000 - 1:15.000), comprende la información registrada de las curvas de nivel en polilínea para la Isla de Cascajal. Estos datos fueron suministrados en formato digital por la Escuela de Arquitectura de la Universidad del Valle (UNIVALLE). La base de datos comprende las curvas de nivel a 2 metros cada una en el formato digital Shapefile con extensión (*.shp).

- Datos de información batimétrica.

- Escala general (1:3.000.000; 1:1.000.000). Comprende la información registrada de las curvas de nivel y los puntos de control para profundidades en la cuenca oceánica del pacífico colombiano. Estos datos fueron suministrados en formato análogo por medio de las cartas náuticas 031 y 032, a través de la Dirección General Marítima (DIMAR). La base de datos comprende las curvas de nivel a 2.000, 1.000, 500, 200 y 100 metros y los puntos de control para las profundidades con valores aleatorios.

- Escala intermedia (1:100.000), comprende la información registrada de las curvas de nivel y los puntos de control para profundidades cercanas a la costa del municipio de Buenaventura. Estos datos fueron suministrados en formato análogo por medio de las cartas náuti- 
cas 150 (Bahía Málaga) y 153 (Golfo de Tortugas) a través de la Dirección General Marítima DIMAR). La base de datos comprende las curvas de nivel a 100, 50, 20 y 10 metros y los puntos de control para las profundidades con valores aleatorios.

- Escala detallada (1:25.000), comprende la información registrada de las curvas de nivel y los puntos de control para las profundidades cercanas a la Bahía de Buenaventura. Estos datos fueron suministrados en formato análogo por medio de la carta náutica 730 (Bahía de Buenaventura) a través de la Dirección General Marítima y la Capitanía de Puerto de Buenaventura. La base de datos comprende las curvas de nivel a 10, 5 y 2 metros y los puntos de control para las profundidades con valores aleatorios.

- Datos de elementos expuestos.

- La información sobre los elementos expuestos poblacional y estructural con sus respectivas variables, fueron obtenidos a partir del Sistema de Consulta de Información Censal - Censo DANE 2005 (Información más reciente a esta escala) (DANE, 2005b).

- Capas vectoriales del departamento del Valle del Cauca con información urbana y estructural. Escala de detalle 1:25.000 (DANE, 2005c).

\section{Determinación de la amenaza sísmica}

El primer paso para la obtención de los valores de amenaza sísmica para el municipio de Buenaventura consiste en el establecimiento de las fuentes sismogénicas por subducción en el pacífico colombiano, dichas unidades sísmicas se comprenden a partir de valores numéricos relacionados con la geometría (coordenadas y profundidad de los vértices) y sismicidad (modelo de ocurrencia sísmica determinado). Para esto, es necesario disponer del registro de eventos sísmicos tsunamigénicos que presenten los parámetros dispuestos.

El registro total de la sismicidad en la región establece tres subfuentes próximas a la zona del litoral pacífico colombiano (SUBN, SUBC y SUBS), comprendidas como segmentos de la fuente inicial (INGEOMINAS - DAGMA, 2005). Las fuentes sísmotectónicas establecidas se representan como estructuras geométricas poligonales (Figura $N^{\circ} 4$ ), cuyo tamaño se expresa por la siguiente relación:

$$
A=K_{1} e^{K_{2} M}
$$

Donde, $A$ es el área de la fuente sísmica $\left(\mathrm{km}^{2}\right), M$ es el registro de magnitudes de eventos sísmicos, y las constantes $K_{1}$ y $K_{2}$, establecen parámetros sobre el radio de ruptura. Dichos parámetros son obtenidos para relación de zonas de ruptura por procesos de subducción establecida por Brune (1970), donde $K_{1}$ y $K_{2}$ toman los valores 0,00381 y 1,15130, respectivamente.

Las coordenadas y valores de profundidad para los vértices que componen cada una de las fuentes, así como el ángulo de buzamiento presente debido a los valores de profundidad, se muestran en el Cuadro $\mathrm{N}^{\circ} 2$. 
Figura $\mathrm{N}^{\circ} 4$

Fuentes sísmicas y eventos sísmicos tsunamigénicos en el occidente colombiano

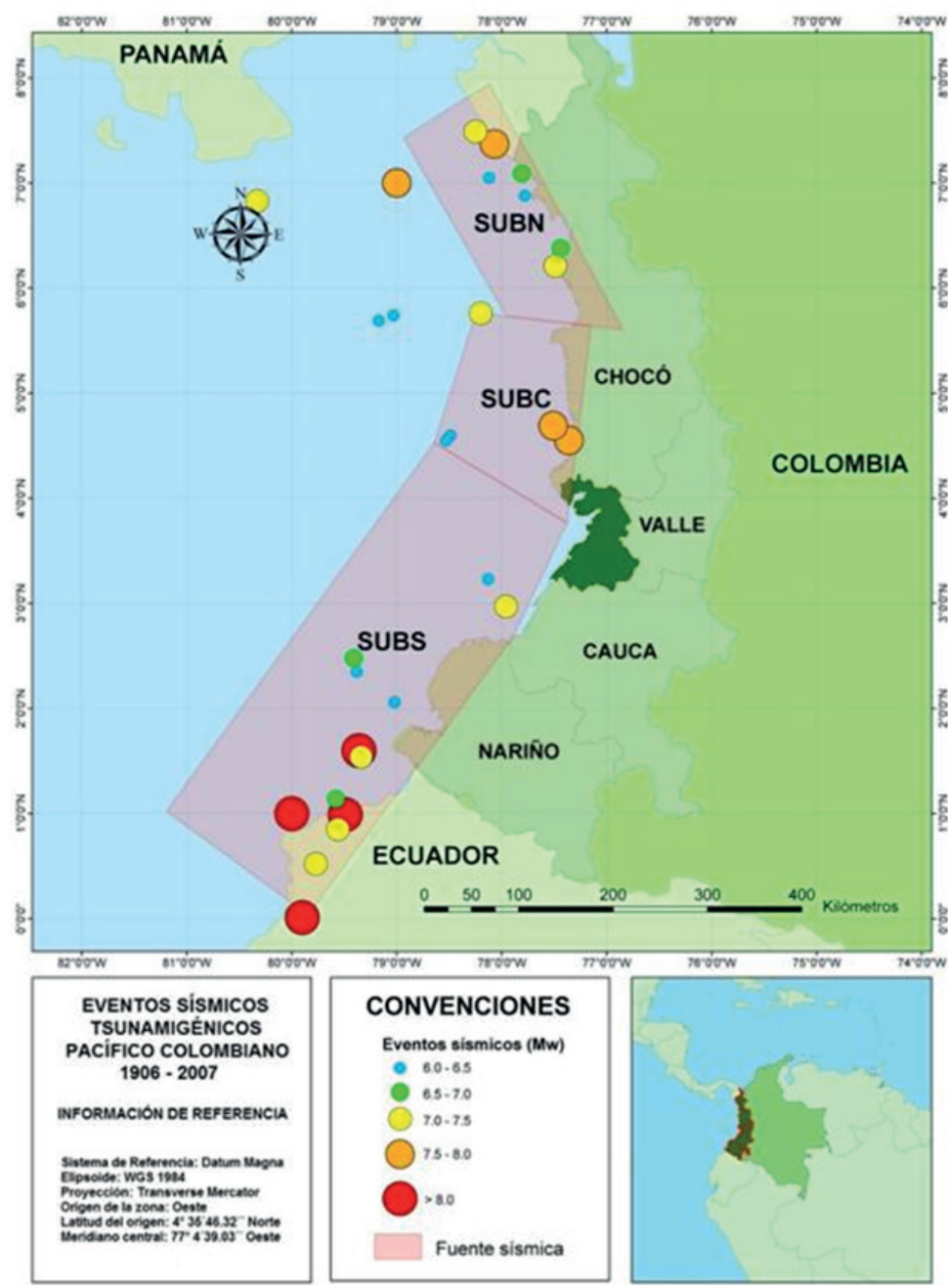

Fuente: Elaboración propia a partir de INGEOMINAS-DAGMA (2005).

Cuadro $\mathrm{N}^{\circ} 2$

Coordenadas de los vértices de las fuentes sísmogénicas consideradas.

\begin{tabular}{|r|r|r|r|r|r|r|r|r|}
\hline \multicolumn{3}{|c|}{ SUBN } & \multicolumn{3}{c|}{ SUBC } & \multicolumn{3}{c|}{ SUBS } \\
\hline Longitud & Latitud & Profundidad & Longitud & Latitud & Profundidad & Longitud & Latitud & Profundidad \\
\hline-78.11 & 7,4 & 50 & -7.71 & 5.64 & 40 & -77.37 & 3.76 & 40 \\
\hline-76.84 & 5.60 & 50 & -77.37 & 3.76 & 40 & -77.89 & 2.68 & 40 \\
\hline-77.95 & 5.73 & 10 & -78.64 & 4.53 & 10 & -79.84 & 0.03 & 40 \\
\hline-78.93 & 7.43 & 10 & -78.24 & 5.76 & 10 & -8.11 & 1.02 & 10 \\
\hline
\end{tabular}


La sismicidad para cada fuente es establecida en función del registro sísmico y la distribución espacio temporal de cada evento al interior de sus límites. Está distribución está asociada a un determinado modelo de ocurrencia sísmica dependiendo del tipo de mecanismo sismogénico de la fuente y la cantidad de información disponible. Por lo tanto, se puede establecer que la sismicidad en un área determinada puede presentar una distribución sísmica del tipo poissoniano (Aleatoria) o de temblor característico (Aguilar, 2001).

Para este estudio se determinó el modelo de ocurrencia del sismo característico para la fuente SUBN (Cuadro $\mathrm{N}^{\circ} 3$ ), ya que los eventos tsunamigénicos son de origen tectónico, pero no presentan una distribución aleatoria con respecto al tiempo, debido a la ausencia de datos disponibles para el establecimiento de una magnitud de umbral. Para las fuentes de subducción SUBC y SUBS se determina el modelo de ocurrencia de Poisson (Cuadro $N^{\circ} 4$ ), debido a que existe la información necesaria para establecer una distribución aleatoria de los eventos tsunamigénicos con respecto al tiempo (Mosquera y Peña, 2010).

Cuadro $\mathrm{N}^{\circ} 3$

Parámetros de sismicidad por sismo característico

\begin{tabular}{|c|c|c|}
\hline \multicolumn{2}{|l|}{ Parámetros } & SUBN \\
\hline \multicolumn{2}{|l|}{ Valor promedio del tiempo entre la ocurrencia de eventos característicos } & 13,25 \\
\hline \multicolumn{2}{|l|}{ Desviación estándar de la magnitud de los eventos característicos } & 13,45 \\
\hline \multicolumn{2}{|l|}{ Magnitud mínima posible de un evento característico } & 6,2 \\
\hline \multicolumn{2}{|l|}{ Magnitud máxima posible de un evento para ser usada en procesos de integración } & 7,7 \\
\hline \multirow{3}{*}{ Parámetros que definen la magnitud esperada como una función del tiempo } & $\mathrm{D}$ & 7,2 \\
\hline & $\mathrm{F}$ & 0 \\
\hline & TOO & 9 \\
\hline
\end{tabular}

Fuente: Cocuñame (2016).

Cuadro $\mathrm{N}^{\circ} 4$

Parámetros de sismicidad por proceso de Poisson

\begin{tabular}{|l|r|r|}
\hline \multirow{2}{*}{ Parámetros } & \multicolumn{2}{|c|}{ Fuente } \\
\cline { 2 - 3 } & \multicolumn{1}{|c|}{ SUBC } & \multicolumn{1}{|c|}{ SUBS } \\
\hline Magnitud de Umbral & 4,0 & 4,0 \\
\hline Tasa de recurrencia $(\lambda)$ & 3,41 & 15,21 \\
\hline Valor esperado de Beta & 0,83 & 0,98 \\
\hline Coeficiente de variación de Beta & 0,03 & 0,03 \\
\hline Magnitud máxima esperada & 7,9 & 8,8 \\
\hline Desviación estándar de la magnitud máxima esperada & 0,1 & 0,1 \\
\hline Límite inferior (M1) & 7,8 & 8,7 \\
\hline Límite superior $(M 2)$ & 8,0 & 8,9 \\
\hline
\end{tabular}

Fuente: Mosquera y Peña (2010). 
Establecidos los parámetros de sismicidad y geometría para las fuentes, el siguiente paso consiste en determinar el modelo de atenuación sísmica correspondiente para cada una de éstas. El modelo de atenuación permite comprender el tipo de desplazamiento de las ondas sísmicas, y los cambios de velocidad de estas, debido a los procesos de absorción física y expansión geométrica debidas a las condiciones geológicas del medio y las alteraciones topográficas en superficie. Los valores de atenuación sísmica utilizados para la zona de estudio se muestran en el Cuadro $N^{\circ} 5$, dichos valores incorporan el registro sísmico en magnitudes, el valor del periodo estructural con un $5 \%$ de amortiguamiento para una ordenada espectral definida (Ordaz, 1997).

\section{Cuadro $N^{\circ} 5$}

Datos de atenuación sísmica

\begin{tabular}{|c|c|c|c|c|c|c|c|c|c|c|c|}
\hline 4 & 8,5 & 10 & \multicolumn{9}{|c|}{$\longleftarrow$ Magnitud mínima, magnitud máxima y $N^{\circ}$ valores por columna } \\
\hline 10 & 200 & 11 & \multicolumn{9}{|c|}{$\longleftarrow$ Distancia mínima, distancia máxima y $\mathrm{N}^{\circ}$ valores por fila } \\
\hline 0.1 & 0.65 & 0 & \multicolumn{9}{|c|}{$\begin{array}{l}\leftarrow \text { Periodo estructural, desviación estándar de la ordenada } \\
\text { espectral y valor máximo posible de intensidad }\end{array}$} \\
\hline 70 & 26 & 13 & 7,5 & 5 & 3,5 & 2,8 & 2,1 & 1,8 & 1,1 & 0,39 & \multirow{10}{*}{ 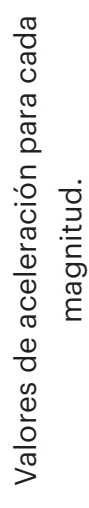 } \\
\hline 140 & 50 & 26 & 17 & 10 & 7,5 & 6 & 5 & 4 & 3,2 & 0,95 & \\
\hline 220 & 90 & 48 & 30 & 20 & 15 & 12 & 9.5 & 8 & 7 & 2 & \\
\hline 360 & 160 & 80 & 50 & 35 & 26 & 20 & 18 & 16 & 14 & 4 & \\
\hline 550 & 240 & 130 & 85 & 60 & 43 & 38 & 30 & 26 & 22 & 8 & \\
\hline 800 & 350 & 200 & 140 & 90 & 70 & 60 & 50 & 42 & 38 & 14 & \\
\hline 1000 & 500 & 300 & 200 & 150 & 110 & 90 & 80 & 70 & 60 & 23 & \\
\hline 1200 & 670 & 400 & 300 & 200 & 170 & 150 & 120 & 110 & 90 & 38 & \\
\hline 1400 & 800 & 520 & 400 & 300 & 220 & 200 & 170 & 160 & 150 & 60 & \\
\hline 1400 & 820 & 600 & 460 & 380 & 300 & 270 & 240 & 210 & 190 & 85 & \\
\hline
\end{tabular}

Fuente: Elaboración propia a partir de Mosquera y Peña, 2010.

Finalmente, se toma en cuenta la información del periodo de retorno, que define el tiempo medio que transcurre entre dos terremotos de magnitud y características similares en un mismo lugar. La siguiente ecuación establece la relación para la obtención de valores de periodo de retorno sísmico:

$$
T=\frac{1}{1-(1-p)^{1 / n}}
$$

Donde, $T$ es el periodo de retorno sísmico (años), $p$ es la probabilidad de ocurrencia de los eventos sísmicos significativos, y $n$ es el tiempo (años) de aquella probabilidad; aunque en temáticas ingenieriles se puede considerar como el tiempo de vida útil de una estructura (SEAOC, 1995).

Para el área de estudio se han establecido cinco valores correspondientes al periodo de retorno, de los cuales cuatro obedecen al tipo de evento telúrico y su relación con la probabilidad de ocurrencia sísmica; mientras que el valor de periodo de retorno restante es considerado a partir de la vida útil de la estructura de hormigón (Cuadro $\mathrm{N}^{\circ}$ 6). 
Cuadro $\mathrm{N}^{\circ} 6$

Datos para los periodos de retorno.

\begin{tabular}{|c|r|r|}
\hline Evento sísmico & Probabilidad de ocurrencia (Años) & Periodo de retorno (Años) \\
\hline Frecuente & 30 & 43 \\
\hline Ocasional & 50 & 72 \\
\hline Raro / Sismo de diseño & 50 & 475 \\
\hline Hormigón (Estructura) & 80 & 760 \\
\hline Muy Raro & 100 & 970 \\
\hline
\end{tabular}

Fuente: SEAOC (1995)

\section{Generación de modelos de elevación digital}

Para la generación de modelos de elevación digital se requiere la información topográfica y batimétrica del área de estudio. Para esto, se llevó a cabo un proceso de adquisición de la información en formatos analógicos y digitales para un posterior procesamiento de los datos e interpolación digital de éstos. El proceso de adquisición de datos define la escala de detalle de la información, el tipo de figura geométrica requerida para el establecimiento de los modelos y el tipo de formato digital o analógico en el que ha sido adquirida. Este conjunto de datos geográficos es representado gráficamente utilizando como ejemplo la carta náutica $N^{\circ} 730$ (Bahía de Buenaventura). En la Figura $N^{\circ} 5$, se muestran los resultados obtenidos tras el proceso de interpolación.

\section{Generación de escenarios de inundación}

Establecidos los modelos de elevación digital y los valores de amenaza sísmica para la zona de estudio se genera el modelo de inundación, el cual muestra en detalle el alcance de las olas para escenarios de inundación probabilísticos determinados por la magnitud del evento sísmico tsunamigénico. El modelo paramétrico de inundación por tsunami establece un número total de 160 epicentros y 1120 eventos sísmicos tsunamigénicos aleatorios. Para dichos eventos sísmicos es necesario identificar aquellos que se consideren como escenarios de amenaza crítica; para esto se ha tomado como principal parámetro de selección la distancia del epicentro a la zona de estudio, y posteriormente la magnitud máxima esperada de dicho epicentro. Este procedimiento se elabora para cada una de las fuentes sísmogénicas establecidas en el análisis de amenaza sísmica (Figura $\mathrm{N}^{\circ} 6$ ), dando como resultado 3 eventos destacados por medio de los cuales se elabora el modelamiento de escenarios de inundación por tsunami para la Isla de Cascajal (Cuadro $\left.N^{\circ} 7\right)$.

Servicio de Impuestos Internos. Tabla de vida útil de los bienes físicos del activo inmovilizado. s/f. Disponible en Internet: http://www.sii.cl/ pagina/valores/bienes/bienes_f.htm 
Figura $\mathrm{N}^{\circ} 5$

Modelos de elevación digital detallados (Isla Cascajal)



Fuente: Elaboración propia.

Cuadro $\mathrm{N}^{\circ} 7$

Parámetros de los eventos tsunamigénicos con amenaza crítica para la Isla de Cascajal

\begin{tabular}{|l|r|r|r|}
\hline Fuente sísmica & \multicolumn{1}{|c|}{ SUBN } & \multicolumn{1}{|c|}{ SUBC } & \multicolumn{1}{c|}{ SUBS } \\
\hline Código de evento & F1 & F11 & F75 \\
\hline Magnitud máxima $(\mathrm{Mw})$ & 7,7 & 7,9 & 8,8 \\
\hline Longitud Oeste & 77,877756 & 77,464303 & 77,526265 \\
\hline Latitud Norte & 5,906986 & 3,987397 & 3,740943 \\
\hline Distancia $(\mathrm{km})$ & 240,65 & 45,36 & 53,44 \\
\hline
\end{tabular}

Fuente: Elaboración propia. 
Figura $N^{\circ} 6$

Modelamiento de eventos tsunamigénicos y modelos elevación digital

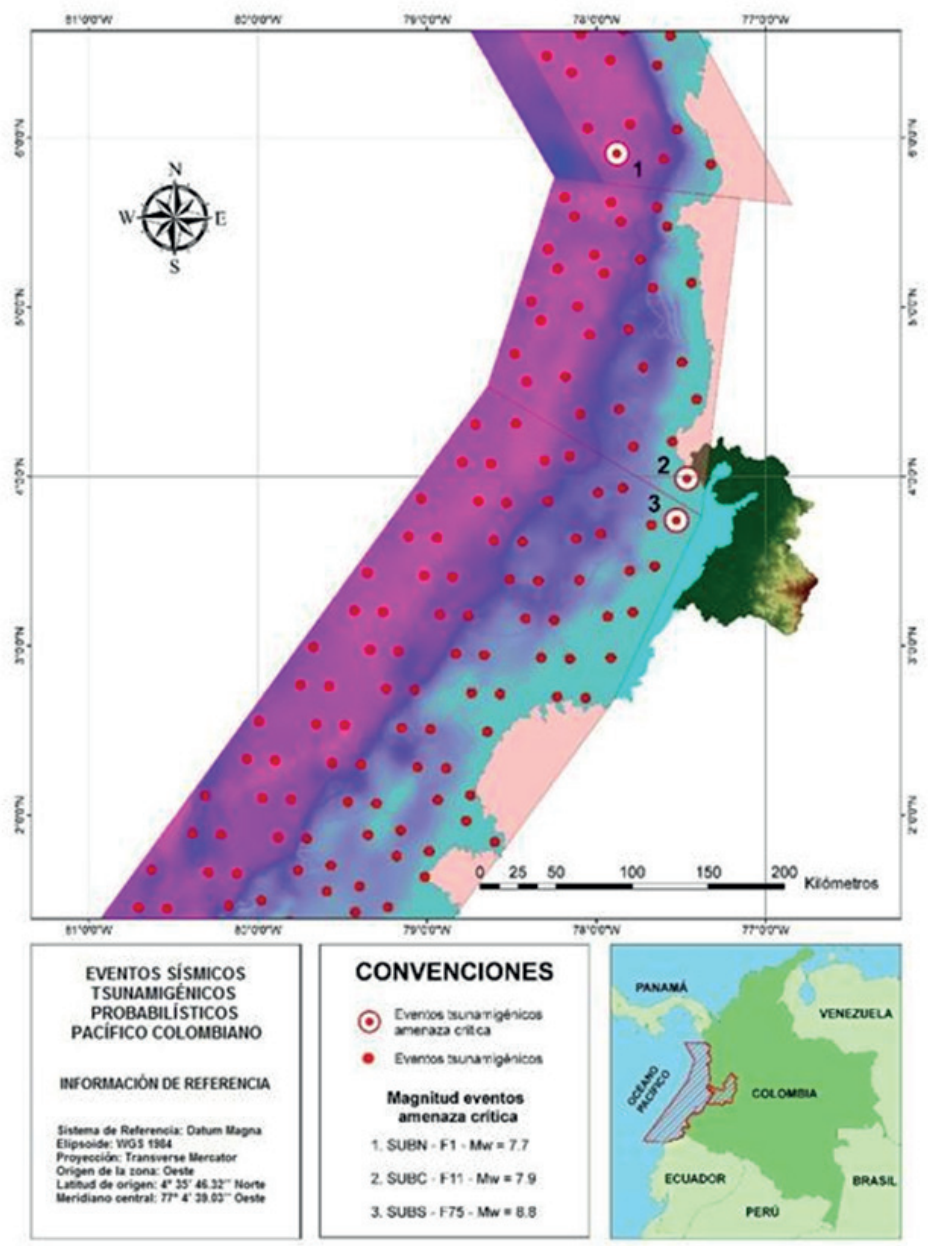

Fuente: Elaboración propia.

Se establecen los escenarios de inundación por tsunami local a partir de los tres eventos sísmicos destacados en el proceso llevado a cabo en este estudio. El tercer escenario de inundación corresponde al evento de mayor amenaza crítica generado por la ocurrencia de un evento sísmico tsunamigénico que tenga lugar en la fuente SUBS con magnitud 8,8 Mw, el cual causaría una inundación que cubriría 3,32 $\mathrm{km}^{2}$ equivalente al 94,6\% del área total de la Isla de Cascajal (Cuadro $\mathrm{N}^{\circ}$ 8, Figura $N^{\circ} 7$ ). La cota máxima hasta donde llega la inundación es de 18,94 metros sobre el nivel del mar, los valores de altitud superiores se encuentran dentro del área no inundable. 
Cuadro $N^{\circ} 8$

Área de inundación por escenario probabilístico (Isla Cascajal).

\begin{tabular}{|c|c|c|c|}
\hline \multicolumn{4}{|c|}{ SUBS F75; Mw: 8,8 } \\
\hline Área & $\mathrm{Km}^{2}$ & $\%$ & Altura (metros) \\
\hline Inundable & 3,32 & 94,6 & \multirow{3}{*}{2,88} \\
\hline No inundable & 0,19 & 5,4 & \\
\hline Total & 3,51 & 100 & \\
\hline
\end{tabular}

Fuente: Elaboración propia.

\section{Análisis de vulnerabilidad}

\section{Determinación de elementos expuestos}

Los elementos expuestos se consideran como aquellos componentes que configuran el escenario de interacciones sociales, económicas, políticas y administrativas en un área específica, y que son susceptibles a verse afectados por una amenaza determinada. Para este estudio se estableció la exposición humana (EH), estructural (ES) y económica (EC), por manzana como la unidad territorial de mayor detalle seleccionada.

La exposición humana (Figura $N^{\circ} 8$ ), se establece a partir de la densidad poblacional, la estructural (Figura $N^{\circ}$ 9) a partir del tipo de vivienda (Casa o apartamento) y la económica (Figura $\mathrm{N}^{\circ} 10$ ) por una sumatoria del número de actividades relacionadas con la industria, comercio y servicios. Las actividades económicas no se especifican en el análisis de vulnerabilidad, debido a la gran cantidad que se consideran en la base de datos del DANE. El Cuadro $N^{\circ}$ 9, establece la cantidad y el porcentaje de elementos expuestos considerados para el análisis de vulnerabilidad humana, estructural y económica, con respecto al área de amenaza de por inundación.

\section{Cuadro $\mathrm{N}^{\circ} 9$}

Relación de elementos y área libre de amenaza.

\begin{tabular}{|l|r|r|r|r|r|}
\hline Elementos expuestos & \multicolumn{1}{|l|}{ Exposición } & \multicolumn{1}{l|}{ \% } & \multicolumn{1}{l|}{ No exposición } & \multicolumn{1}{l|}{ Total } \\
\hline Humano (Población) & 60.339 & 75,06 & 20.039 & 24,94 & 80.378 \\
\hline Estructural (Viviendas) & 12.514 & 75,9 & 3.973 & 24,1 & 16.487 \\
\hline Económico (Actividades) & 2.198 & 69,7 & 952 & 30,3 & 3.150 \\
\hline
\end{tabular}

Fuente: Elaboración propia. 
Figura $\mathrm{N}^{\circ} 7$

Escenario de inundación en la Isla de Cascajal, municipio de Buenaventura, considerando un posible evento sísmico tsunamigénico de magnitud $8.8 \mathrm{Mw}$ en la fuente SUBS
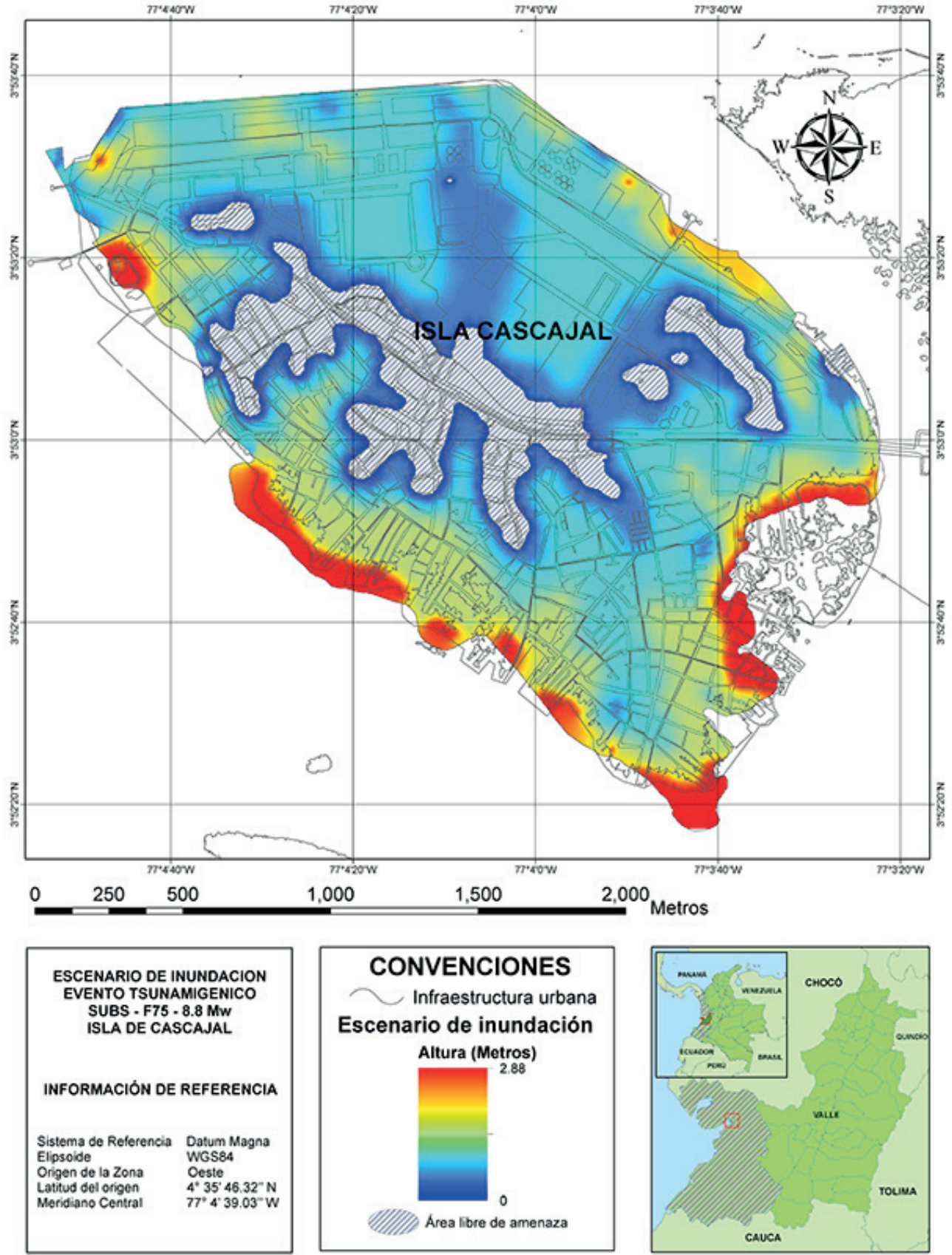

Fuente: Elaboración propia. 
Figura $\mathrm{N}^{\circ} 8$

Exposición humana por densidad poblacional

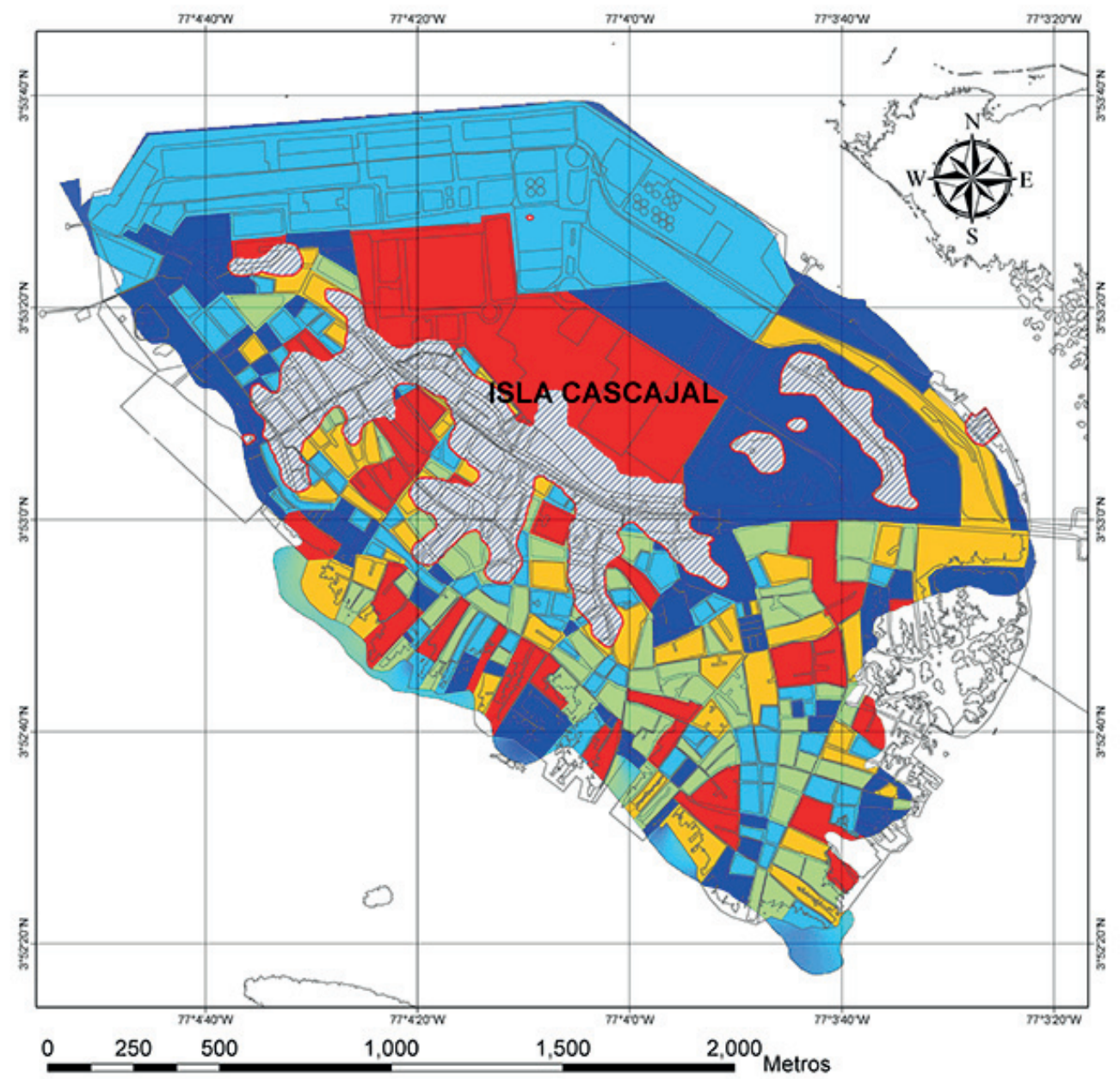

\begin{tabular}{|c|c|}
\hline \multicolumn{2}{|c|}{$\begin{array}{l}\text { DENSIDAD POBLACIONAL } \\
\text { UNIDAD TERRITORIAL } \\
\text { MANZANA }\end{array}$} \\
\hline \multicolumn{2}{|c|}{$\begin{array}{l}\text { EXPOSICIÓN } \\
\text { ANTE EVENTO TSUNAMIGÉNICO } \\
\text { SUBS - F75 - } 8.8 \mathrm{Mw} \\
\text { ISLA DE CASCAJAL }\end{array}$} \\
\hline \multicolumn{2}{|c|}{ INFORMACIÓN DE REFERENCIA } \\
\hline $\begin{array}{l}\text { Sistema de Referencia } \\
\text { Elipsoide } \\
\text { Origen de la Zona } \\
\text { Latitud del origen } \\
\text { Meridiano Central }\end{array}$ & $\begin{array}{l}\text { Datum Magna } \\
\text { WGS84 } \\
\text { Oeste } \\
4^{*} 35^{\prime} 46.32^{-1} \mathrm{~N} \\
77^{*} 4^{\prime} 39.03^{-1} \mathrm{~W}\end{array}$ \\
\hline
\end{tabular}



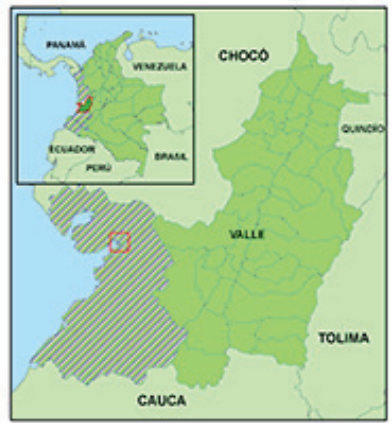

Fuente: Elaboración propia. 
Figura $N^{\circ} 9$

Exposición estructural, a partir del tipo de vivienda predominante por manzanas
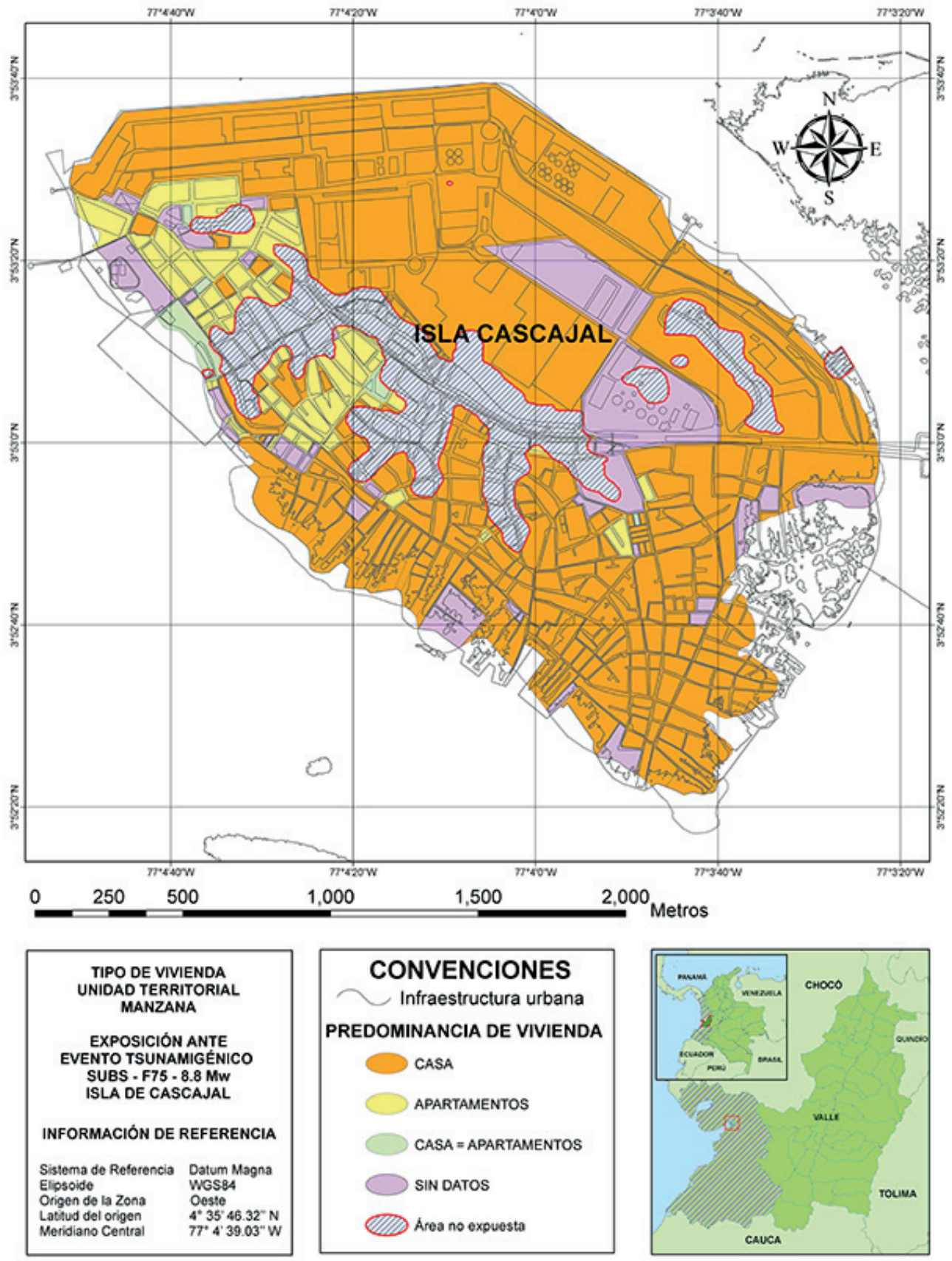

Fuente: Elaboración propia. 
Figura $N^{\circ} 10$

Exposición económica, a partir del tipo de la sumatoria de actividades por manzana

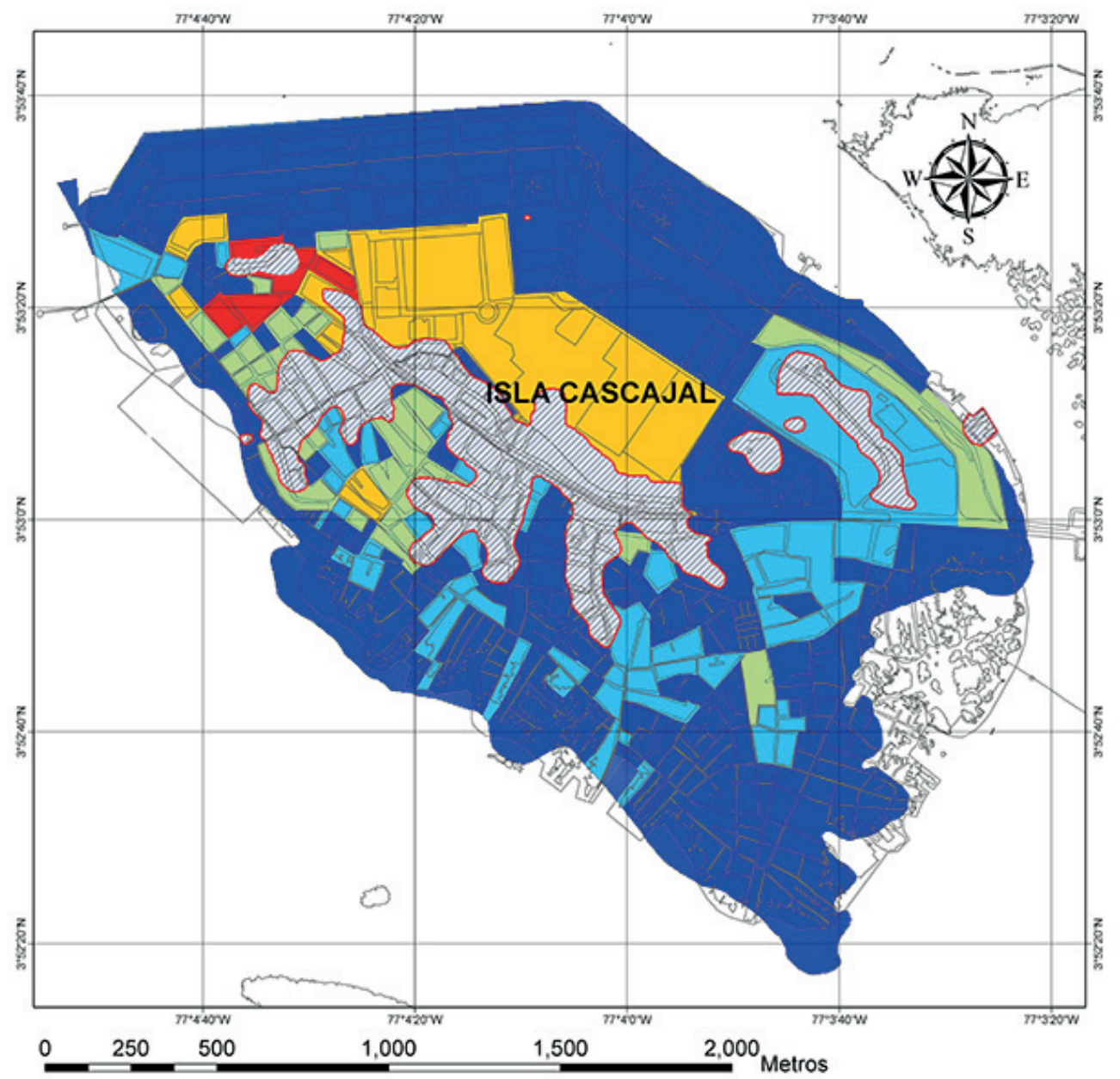

\begin{tabular}{|c|c|}
\hline \multicolumn{2}{|c|}{$\begin{array}{l}\text { ACTIVIDADES ECONÓMICAS } \\
\text { INDUSTRIA } \\
\text { COMERCIO } \\
\text { SERVICIOS }\end{array}$} \\
\hline \multicolumn{2}{|c|}{$\begin{array}{l}\text { EXPOSICION ANTE } \\
\text { EVENTO TSUNAMIGÉNICO } \\
\text { SUBS - F75 }-8.8 \mathrm{MW} \\
\text { ISLA DE CASCAJAL }\end{array}$} \\
\hline \multicolumn{2}{|c|}{ INFORMACIÓN DE REFERENCIA } \\
\hline $\begin{array}{l}\text { Sistema de Referencia } \\
\text { Elipsoide } \\
\text { Origen de la Zona } \\
\text { Lathud del origen } \\
\text { Meridiano Central }\end{array}$ & $\begin{array}{l}\text { Datum Magna } \\
\text { WGS84 } \\
\text { Oeste } \\
4^{*} 35^{\circ} 46.32^{-1} \mathrm{~N} \\
77^{*} 4^{\prime} 39.03^{\prime \prime} \mathrm{W}\end{array}$ \\
\hline
\end{tabular}



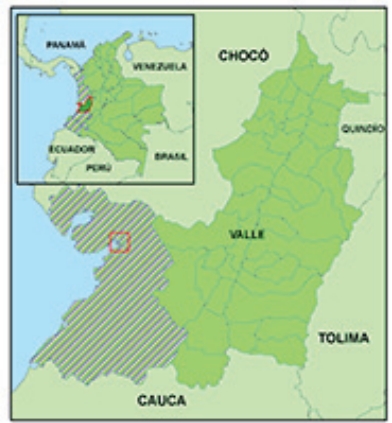

Fuente: Elaboración propia. 


\section{Fragilidad de los elementos expuestos}

Para cada uno de los elementos expuestos en el área de inundación para la Isla de Cascajal, establecida por el escenario tsunamigénico de amenaza crítica, existen condiciones específicas que los ponen en mayor situación de vulnerabilidad frente impacto de la amenaza mencionada.

Para los elementos expuestos poblacionales, se han considerado las siguientes características de las personas que conllevarían al aumento de la vulnerabilidad a través del grado de fragilidad humana:

- Edad: La población del casco urbano de la Isla de Cascajal que se encuentre dentro de los siguientes intervalos de edad:

$$
9>\operatorname{Edad}(\text { Años })>60
$$

Este grupo se considera altamente vulnerable ante una emergencia de carácter natural o antrópico, debido a la capacidad de respuesta ante estas, la velocidad de desplazamiento, el conocimiento del entorno y otro tipo de variables (Figura $\left.\mathrm{N}^{\circ} 11\right)$.

- Limitaciones: La cantidad de personas que presenten alguna discapacidad de carácter psicológico o motriz hacen que se vean limitadas a responder efectivamente en caso de presentarse una emergencia por inundación (Figura $\mathrm{N}^{\circ} 12$ ).

Para la Exposición Estructural y Ecónomica, se han considerado dos características que aumentan la vulnerabilidad en las edificaciones y las actividades económicas circunscritas en el área:

- Tipo de vivienda: En el presente estudio se establecen dos tipos de edificaciones que componen los elementos estructurales, casas y apartamentos. De ellos, las casas presentan un mayor grado de fragilidad debido su altura respecto a la cota de inundación. Mientras que los apartamentos, a partir del segundo piso tendrán menor contacto con el área de inundación (Figura $\mathrm{N}^{\circ} 13$ ).

- Cobertura de servicios públicos: La red de servicios públicos considerados para este análisis contempla el suministro de energía eléctrica, alcantarillado y acueducto, así como el servicio telefónico. Un mayor porcentaje en la disponibilidad de estos servicios genera mayor condición de vulnerabilidad en las manzanas y unidades territoriales emplazadas dentro del área de estudio (Figura $\mathrm{N}^{\circ} 14$ ).

La estimación de cada uno de estas características de fragilidad se establece a través del promedio total de los casos por manzana, y la posterior interpolación de esta información a través del método geoestadístico Kriging, el cual se encuentra disponible dentro de las plataformas de geoprocesamiento de ArcGIS. 
Figura No 11

Fragilidad humana a partir del porcentaje de personas vulnerables por intervalo de edad

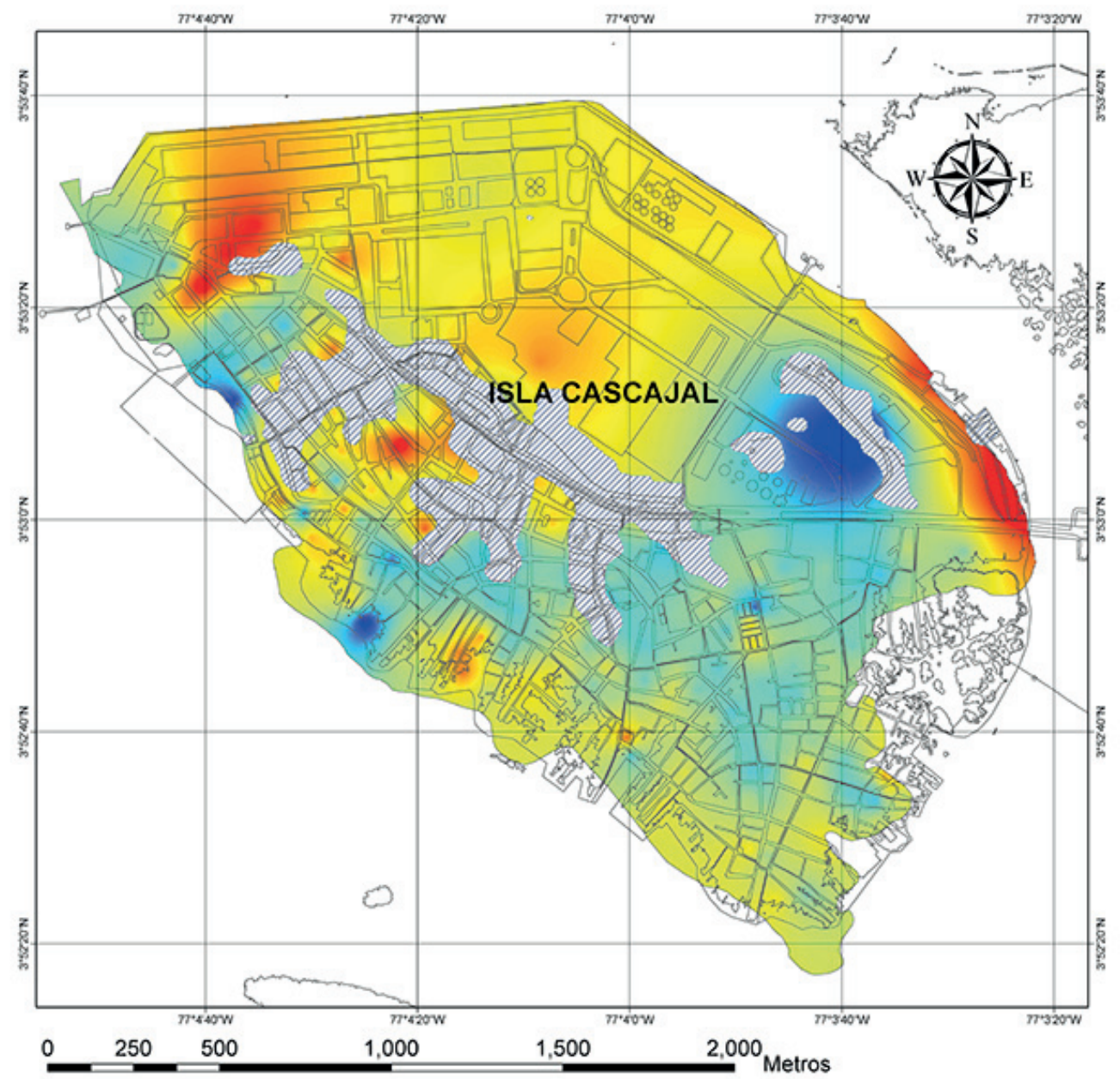

\begin{tabular}{|c|c|}
\hline \multicolumn{2}{|c|}{$\begin{array}{c}\text { POBLACIÓN FRÁGIL } \\
\text { POR INTÉRVALOS DE EDAD } \\
\text { OA } 9 \text { AN̂OS } \\
\text { MAYORES DE } 60 \text { AÑOS }\end{array}$} \\
\hline \multicolumn{2}{|c|}{$\begin{array}{l}\text { EXPOSICIÓN ANTE } \\
\text { EVENTO TSUNAMIGÉNICO } \\
\text { SUBS - F75 - } 8.8 \mathrm{MW} \\
\text { ISLA DE CASCAJAL }\end{array}$} \\
\hline \multicolumn{2}{|c|}{ INFORMACIÓN DE REFERENCIA } \\
\hline $\begin{array}{l}\text { Sistema de Referencia } \\
\text { Elipsoide } \\
\text { Origen de la Zona } \\
\text { Lathud del origen } \\
\text { Meridiano Central }\end{array}$ & $\begin{array}{l}\text { Datum Magna } \\
\text { WGS84 } \\
\text { Oeste } \\
4^{+} 35^{\circ} 46.32^{-} \mathrm{N} \\
7^{*} 4^{\prime} 39.03^{\prime \prime} \mathrm{W}\end{array}$ \\
\hline
\end{tabular}


Fuente: Elaboración propia. 
Figura $\mathrm{N}^{\circ} 12$

Fragilidad humana a partir del porcentaje de población con limitaciones o discapacidades


Fuente: Elaboración propia. 
Figura $\mathrm{N}^{\circ} 13$

Fragilidad estructural a partir del porcentaje de casas presente por manzana

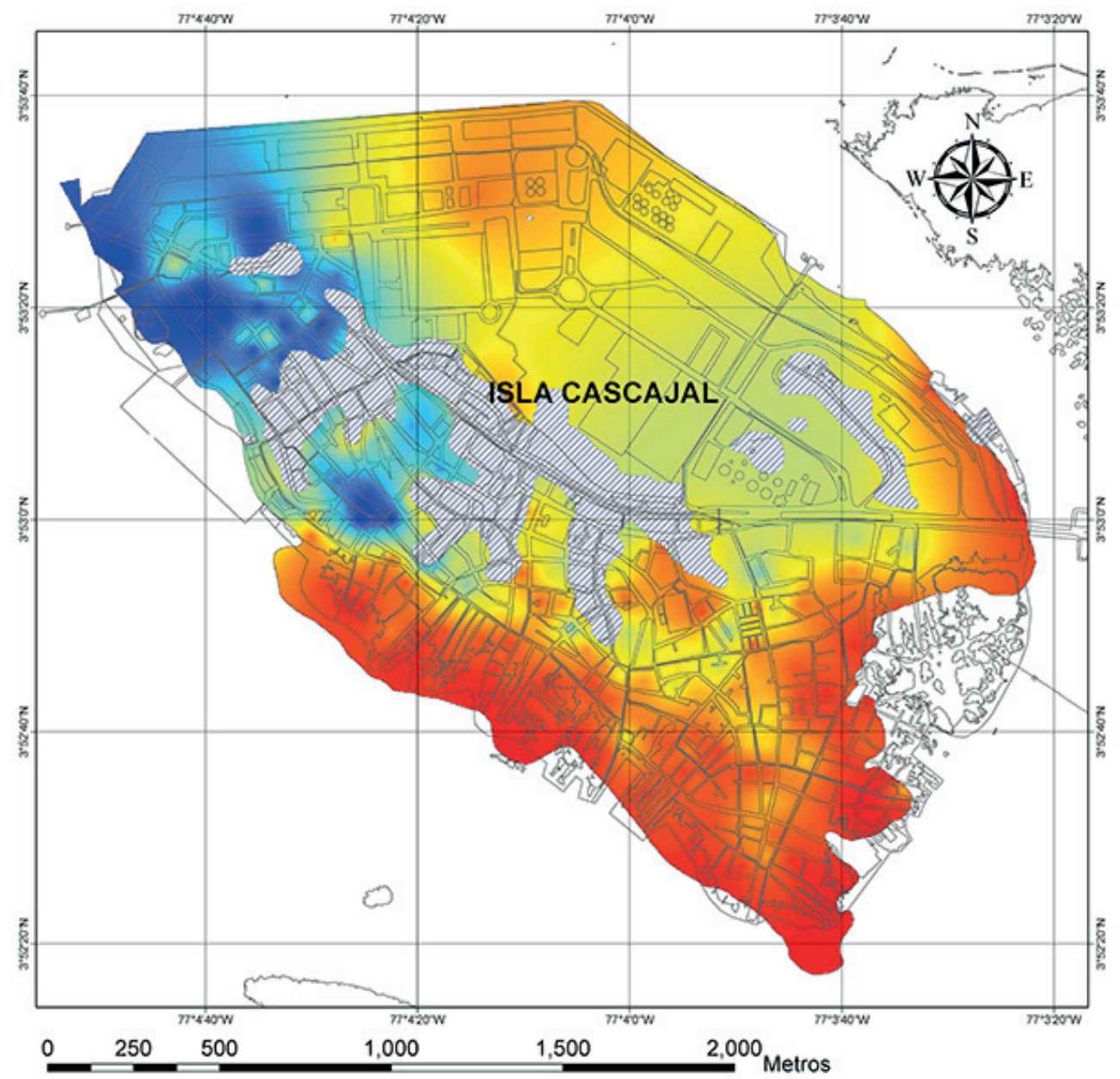

\begin{tabular}{|c|c|}
\hline \multicolumn{2}{|c|}{$\begin{array}{l}\text { FRAGILIDAD ESTRUCTURAL } \\
\text { TIPO DE VIVIENDA } \\
\text { CASAS Y APARTAMENTOS }\end{array}$} \\
\hline \multicolumn{2}{|c|}{$\begin{array}{l}\text { EXPOSICIÓN ANTE } \\
\text { EVENTO TSUNAMIGÉNICO } \\
\text { SUBS - F75 - } 8.8 \mathrm{MW} \\
\text { ISLA DE CASCAJAL }\end{array}$} \\
\hline \multicolumn{2}{|c|}{ INFORMACIÓN DE REFERENCIA } \\
\hline $\begin{array}{l}\text { Sistema de Referencia } \\
\text { Elipsoide } \\
\text { Origen de la Zona } \\
\text { Lathud del origen } \\
\text { Meridiano Central }\end{array}$ & $\begin{array}{l}\text { Datum Magna } \\
\text { WGSe4 } \\
\text { Oeste } \\
4^{*} 35^{\circ} 46.32^{\prime \prime} \mathrm{N} \\
7^{*} 4^{\prime} 39.03^{\prime \prime} \mathrm{W}\end{array}$ \\
\hline
\end{tabular}
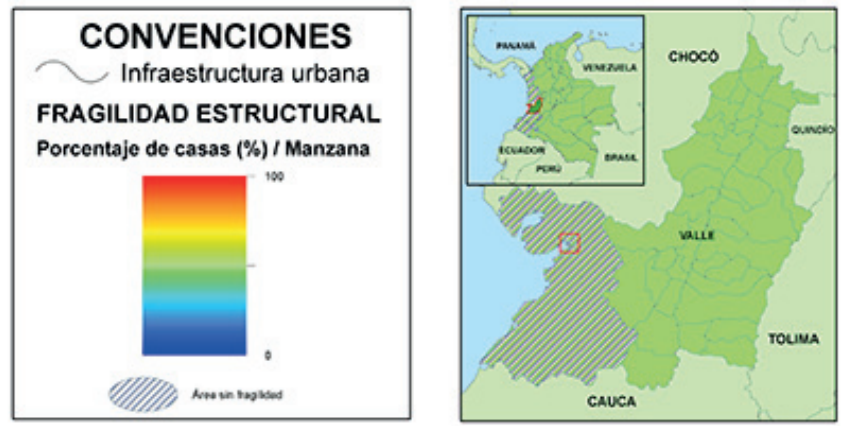

Fuente: Elaboración propia. 
Figura $\mathrm{N}^{\circ} 14$

Fragilidad a partir de porcentaje de cobertura en los servicios públicos por manzana.

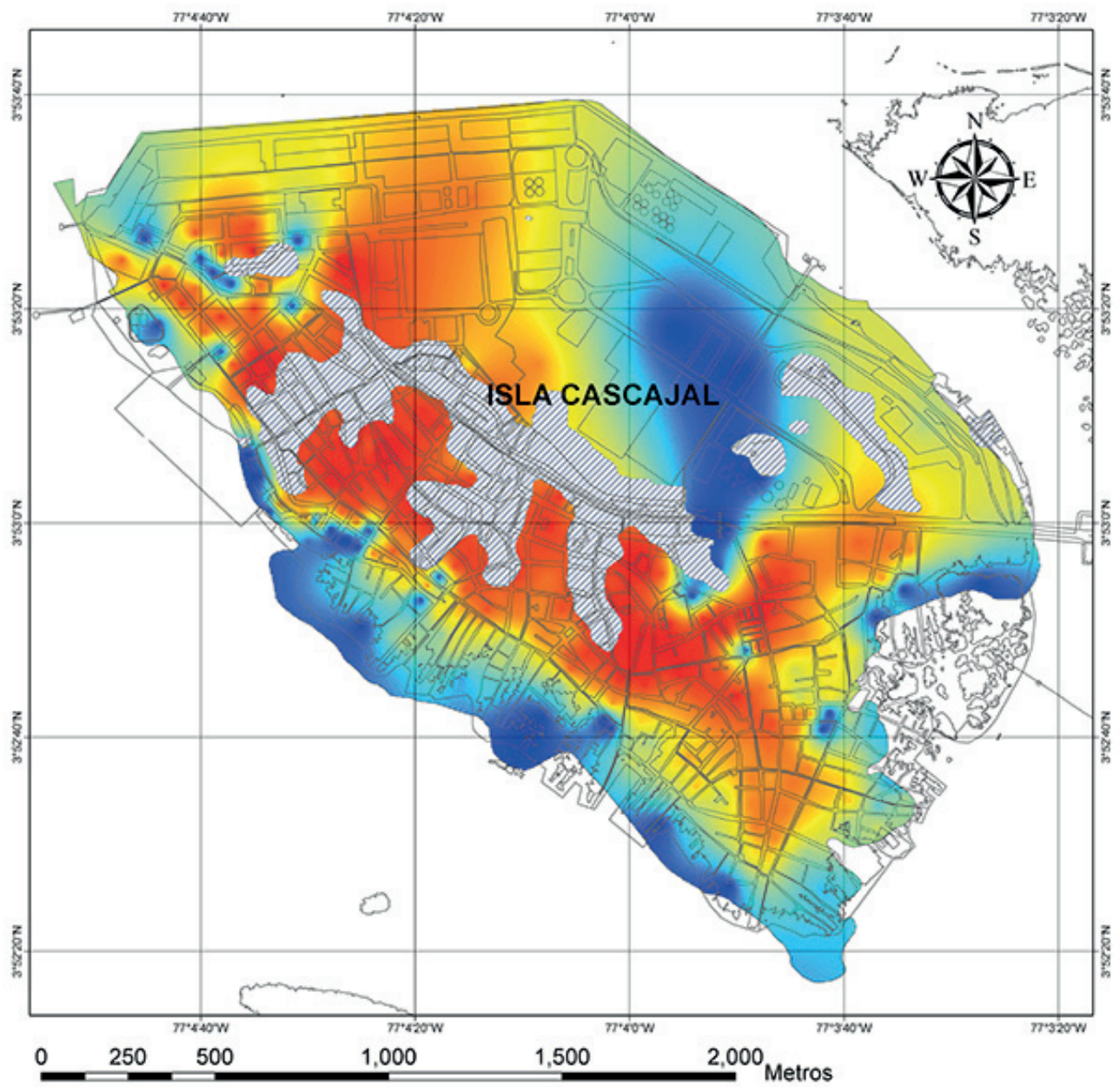

\begin{tabular}{|c|c|}
\hline \multicolumn{2}{|c|}{$\begin{array}{c}\text { FRAGILIDAD ESTRUCTURAL } \\
\text { COBERTURA SERVICIOS PÚBLICOS } \\
\text { ELECTRICIDAD, ALCANTARILLADO } \\
\text { ACUEDUCTO, TELÉFONO }\end{array}$} \\
\hline $\begin{array}{l}\text { EXPOSICIÓN } \\
\text { EVENTO TSUNA } \\
\text { SUBS - F75 - } \\
\text { ISLA DE CAS }\end{array}$ & $\begin{array}{l}\text { ANTE } \\
\text { MIGÉNICO } \\
8.8 \mathrm{MW} \\
\text { CAJAL }\end{array}$ \\
\hline INFORMACIÓN DE & REFERENCIA \\
\hline $\begin{array}{l}\text { Sistema de Referencia } \\
\text { Elipsoide } \\
\text { Origen de la Zona } \\
\text { Latitud del origen } \\
\text { Meridiano Central }\end{array}$ & $\begin{array}{l}\text { Datum Magna } \\
\text { WGS84 } \\
\text { Oeste } \\
4^{*} 35^{\prime} 46.32^{\prime \prime} \mathrm{N} \\
77^{*} 4^{\prime} 39.03^{\prime \prime} \mathrm{W}\end{array}$ \\
\hline
\end{tabular}
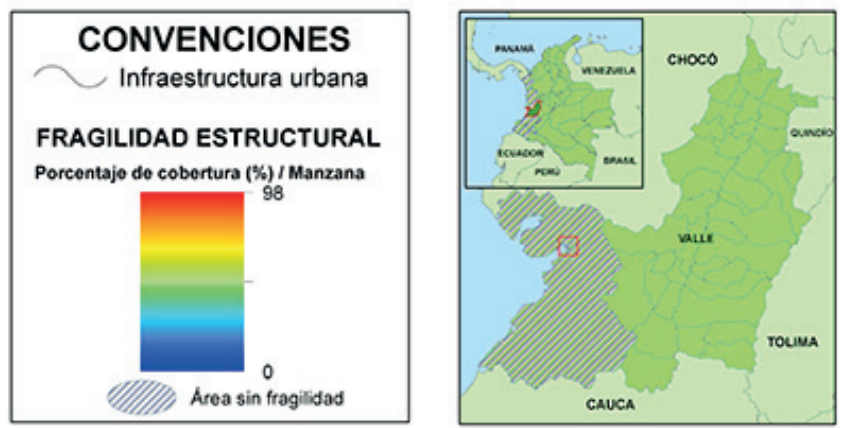

Fuente: Elaboración propia. 


\section{Cálculo de la vulnerabilidad}

La clasificación de los grados de vulnerabilidad tanto humana como estructural y económica se lleva a cabo a partir de un proceso de interpolación de los datos porcentuales de los factores de fragilidad, dentro de los elementos expuestos localizados al interior del área de estudio. Para este análisis se han considerado tres grados de vulnerabilidad que van desde el nivel Alto (Rojo), medio (Verde) y bajo (Azul), indicando que la población (Figura $N^{\circ} 15$ ) que se encuentra dentro de aquellas unidades poligonales, así como los equipamientos y estructuras (Figura $N^{\circ} 16$ ), presentan una menor capacidad de respuesta y mayor exposición por fragilidad ante el escenario de amenaza crítica establecido en el presente estudio.

El Cuadro $N^{\circ} 10$ establece los valores de vulnerabilidad obtenidos con respecto al escenario de amenaza de inundación crítica, previamente mencionado.

Cuadro $N^{\circ} 10$

Relación de vulnerabilidad para los elementos expuestos.

\begin{tabular}{|l|r|r|r|r|r|r|r|}
\hline $\begin{array}{l}\text { Elementos } \\
\text { expuestos }\end{array}$ & $\begin{array}{c}\text { Vulnerabilidad } \\
\text { (Alta) }\end{array}$ & $\%$ & $\begin{array}{c}\text { Vulnerabilidad } \\
\text { (Media) }\end{array}$ & $\%$ & $\begin{array}{c}\text { Vulnerabilidad } \\
\text { (Baja) }\end{array}$ & $\%$ & Total \\
\hline $\begin{array}{l}\text { Humano } \\
\text { (Población) }\end{array}$ & 7.512 & 12,45 & 36.836 & 61,05 & 15.991 & 26,5 & 60.339 \\
\hline $\begin{array}{l}\text { Estructural } \\
\text { Económico }\end{array}$ & 10.279 & 69,87 & 2.309 & 15,7 & 2.124 & 14,43 & 14.712 \\
$\begin{array}{l}\text { (Viviendas } \\
\text { Actividades) }\end{array}$ & & & & & & \\
\hline
\end{tabular}

Fuente: Elaboración propia. 
Figura $\mathrm{N}^{\circ} 15$

Vulnerabilidad humana a partir de elementos expuestos y su fragilidad

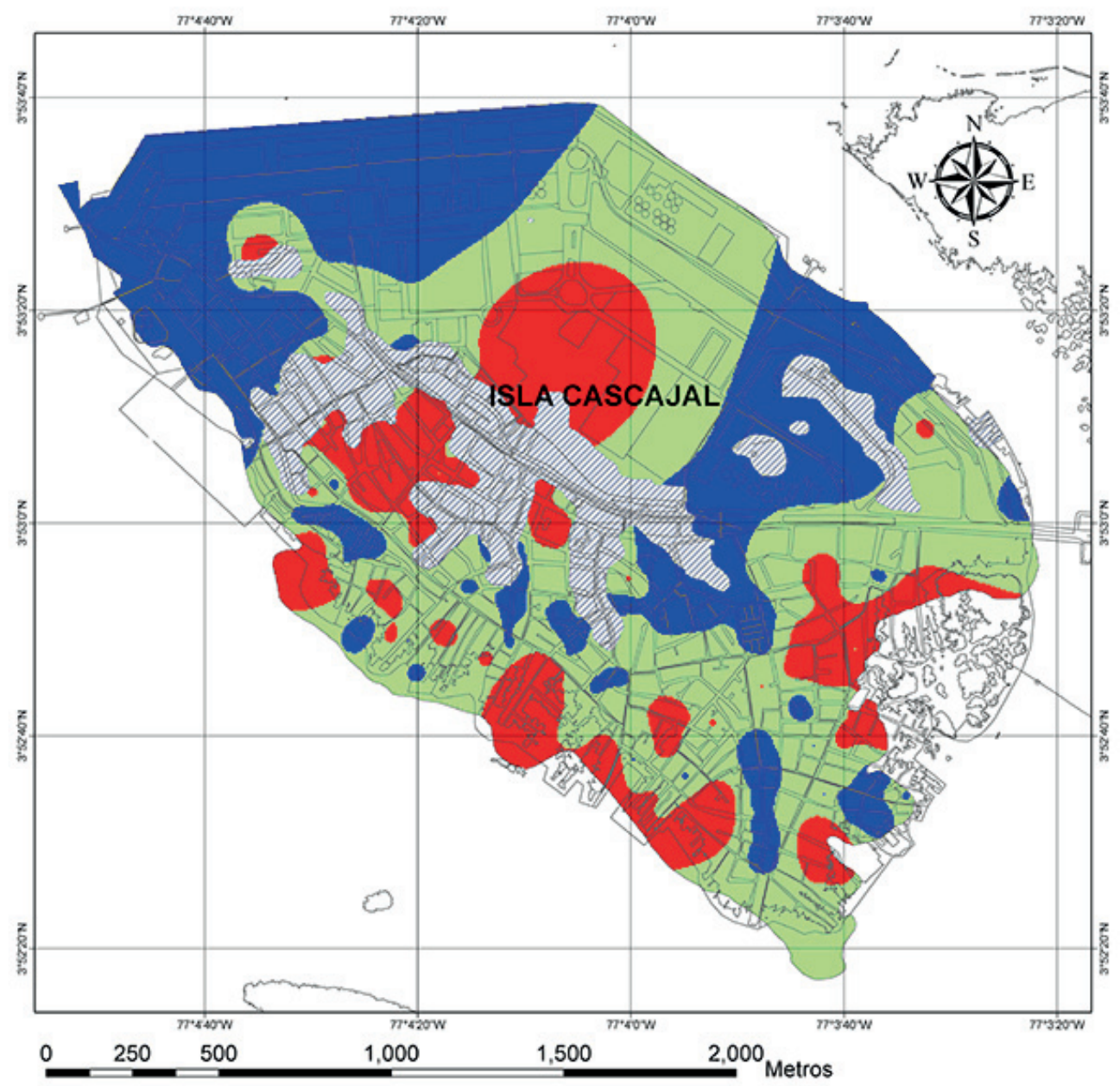

\begin{tabular}{|c|c|}
\hline \multicolumn{2}{|c|}{$\begin{array}{l}\text { VULNERRABILIDAD HUMANA } \\
\text { POBLACIÓN FRAGIL } \\
\text { INTÉRVALOS DE EDAD } \\
\text { LIMITACIONES O DISCAPACIDAD }\end{array}$} \\
\hline \multicolumn{2}{|c|}{$\begin{array}{l}\text { EXPOSICIÓN ANTE } \\
\text { EVENTO TSUNAMIGÉNICO } \\
\text { SUBS - F75 - } 8.8 \mathrm{Mw} \\
\text { ISLA DE CASCAJAL }\end{array}$} \\
\hline \multicolumn{2}{|c|}{ INFORMACIÓN DE REFERENCIA } \\
\hline $\begin{array}{l}\text { Sistema de Referencia } \\
\text { EElpsoide } \\
\text { Origen de la Zona } \\
\text { Latudud del origen } \\
\text { Meridiano Central }\end{array}$ & $\begin{array}{l}\text { Datum Magna } \\
\text { WGSB4 } \\
\text { Oeste } \\
4^{*} 35^{4} 46.32^{-2} \mathrm{~N} \\
77^{4} 4^{4} 39.03^{\prime \prime} \mathrm{W}\end{array}$ \\
\hline
\end{tabular}
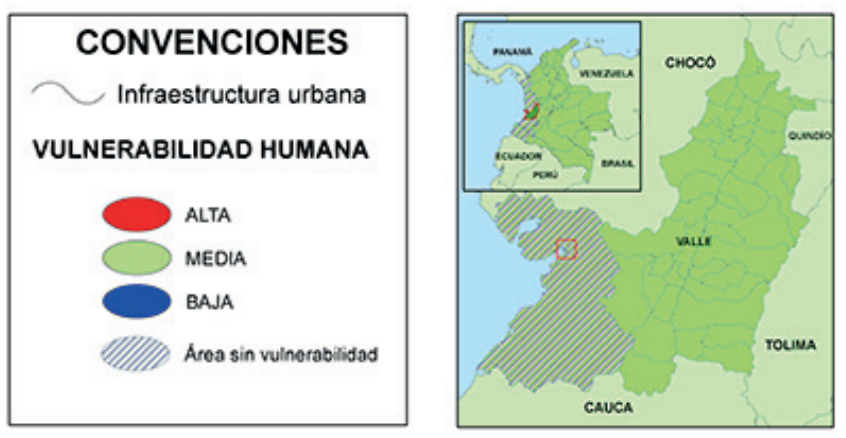

Fuente: Elaboración propia. 
Figura $\mathrm{N}^{\circ} 16$

Vulnerabilidad estructural y económica a partir de elementos expuestos y fragilidad

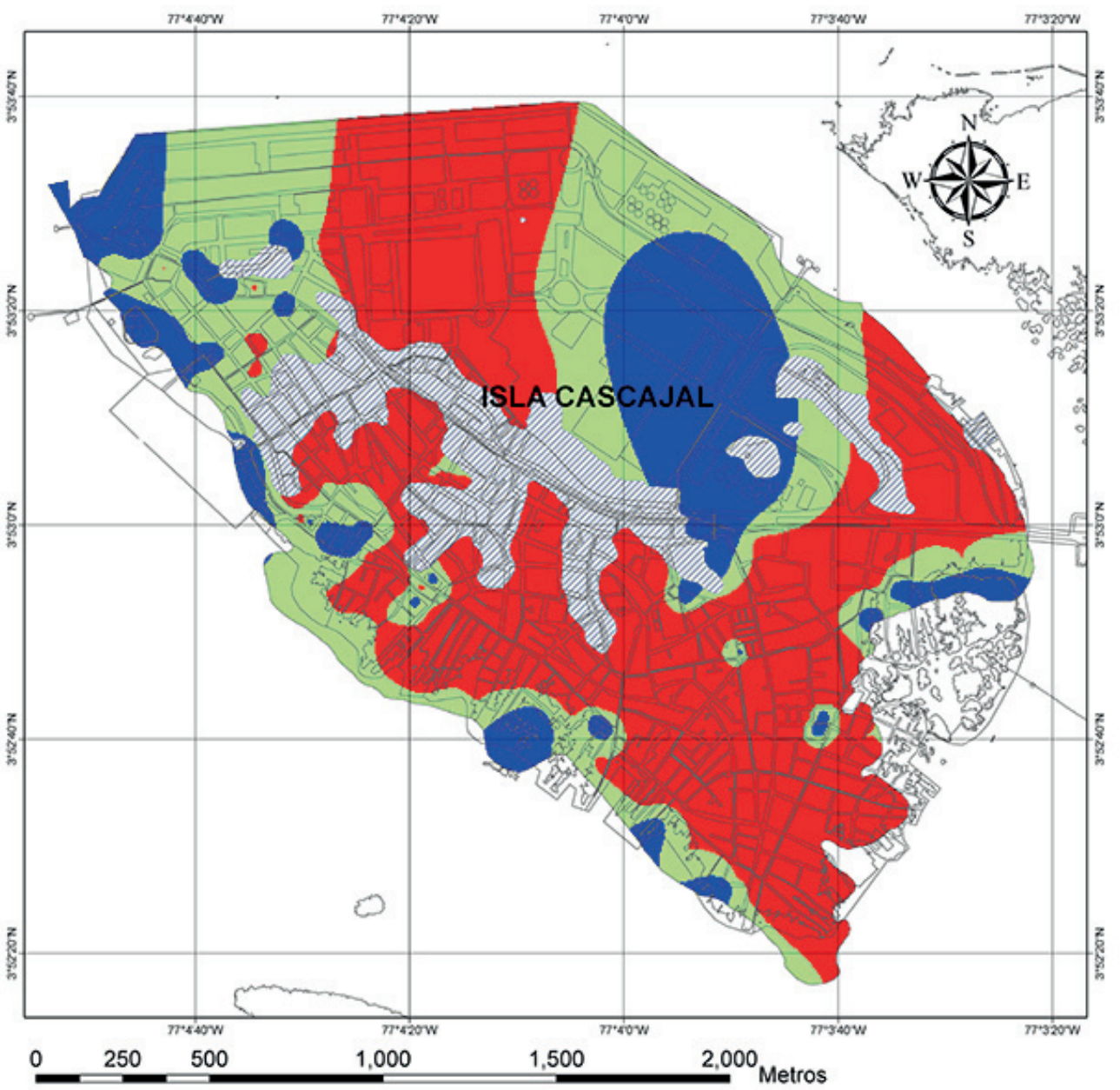

\begin{tabular}{|c|c|}
\hline \multicolumn{2}{|c|}{$\begin{array}{l}\text { VULNERABILIDAD } \\
\text { ESTRUCTURAL Y ECONOMICA } \\
\text { FRAGILIDAD POR } \\
\text { TIPO DE VIVIENDA } \\
\text { COBERTURA SERVICIOS PÚBLICOS }\end{array}$} \\
\hline \multicolumn{2}{|c|}{$\begin{array}{l}\text { EXPOSICION ANTE } \\
\text { EVENTO TSUNAMIGENICO } \\
\text { SUBS - F75 - } 8.8 \mathrm{MW} \\
\text { ISLA OE CASCAJAL }\end{array}$} \\
\hline \multicolumn{2}{|c|}{ INFORMACIÓN DE REFERENCIA } \\
\hline $\begin{array}{l}\text { Sistema de Referencia } \\
\text { Elipsoide } \\
\text { Origen de la Zona } \\
\text { Lathud del origen } \\
\text { Meridiano Central }\end{array}$ & $\begin{array}{l}\text { Datum Magna } \\
\text { WGS } \\
\text { Oeste } \\
4^{*} 35^{\circ} 46.32^{-1} \mathrm{~N} \\
77^{*} 4^{\prime} 39.03^{-1} \mathrm{~W}\end{array}$ \\
\hline
\end{tabular}
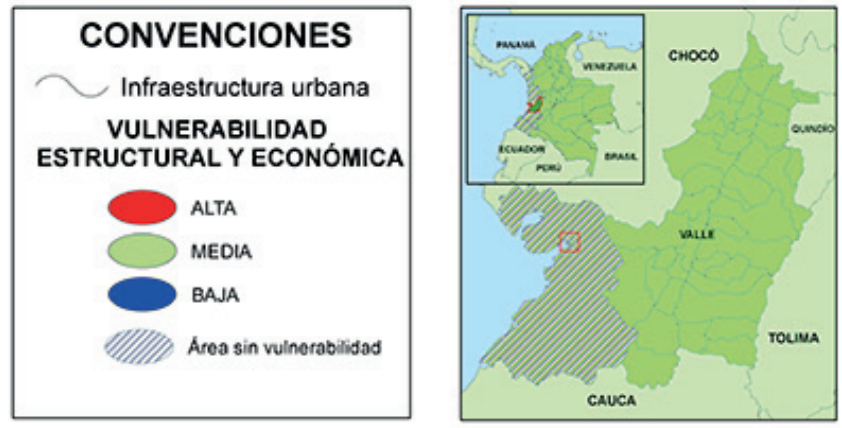

Fuente: Elaboración propia. 


\section{Evaluación del riesgo}

\section{Afectación de elementos expuestos}

Finalmente, se establece la caracterización de zonas de alto, medio y bajo riesgo relacionados con el impacto del evento sísmico tsunamigénico de orden probabilístico, relacionando la cantidad de afectación para los elementos expuestos dentro del área de amenaza crítica.

Los resultados obtenidos indican que, para el componente de vulnerabilidad humana, la población presente dentro del área de estudio no se encuentra dentro del intervalo de edad considerado frágil como tampoco cubre un porcentaje considerable de personas con limitaciones psicológicas y motrices, lo que permite definir que el riesgo de afectación de la población civil sea medio y bajo (Figura $N^{\circ} 17$ ).

Para el componente de vulnerabilidad estructural y económica, los resultados indican mayor propensión a existir afectaciones estructurales debido al alto porcentaje de viviendas tipo casa y la presencia de una alta cobertura en servicios públicos suministrados de manera directa e indirecta por las autoridades locales y departamentales (Figura $N^{\circ} 18$ ).

Las principales afectaciones se concentran al sur de la Isla de Cascajal, donde se encuentran emplazados los sectores de menor desarrollo económico y social del municipio, mientras que el sector norte (instalaciones portuarias) presentan menor grado de riesgo en el componente humano, en contraste con un alto grado de afectación debido a la presencia de los equipamientos inmobiliarios y la cobertura de servicios públicos.

La afectación de los elementos expuestos se encuentra detallada en el Cuadro $N^{\circ} 11$.

\section{Cuadro $\mathrm{N}^{\circ} 11$}

Resultados porcentuales del nivel de riesgo para los elementos expuestos.

\begin{tabular}{|l|r|r|r|r|r|r|r|}
\hline $\begin{array}{l}\text { Elementos } \\
\text { expuestos }\end{array}$ & $\begin{array}{c}\text { Riesgo } \\
\text { (Alto) }\end{array}$ & $\%$ & $\begin{array}{l}\text { Riesgo } \\
\text { (Media) }\end{array}$ & \multicolumn{1}{c|}{$\%$} & \multicolumn{1}{c|}{$\begin{array}{c}\text { Riesgo } \\
\text { (Baja) }\end{array}$} & \multicolumn{1}{c|}{$\%$} & Total \\
\hline $\begin{array}{l}\text { Humano } \\
\text { (Población) }\end{array}$ & 5.792 & 9,6 & 48.005 & 79,56 & 6.540 & 10,84 & 60.339 \\
\hline $\begin{array}{l}\text { Estructural } \\
\text { Económico }\end{array}$ & 12.269 & 83,4 & 1.250 & 8,5 & 1.191 & 8,1 & 14.712 \\
$\begin{array}{l}\text { Viviendas } \\
\text { Actividades } \\
\text { Servicios } \\
\text { públicos }\end{array}$ & & & & & & & \\
\hline
\end{tabular}

Fuente: Elaboración propia. 
Figura $\mathrm{N}^{\circ} 17$

Riesgo de inundación a partir de la exposición humana

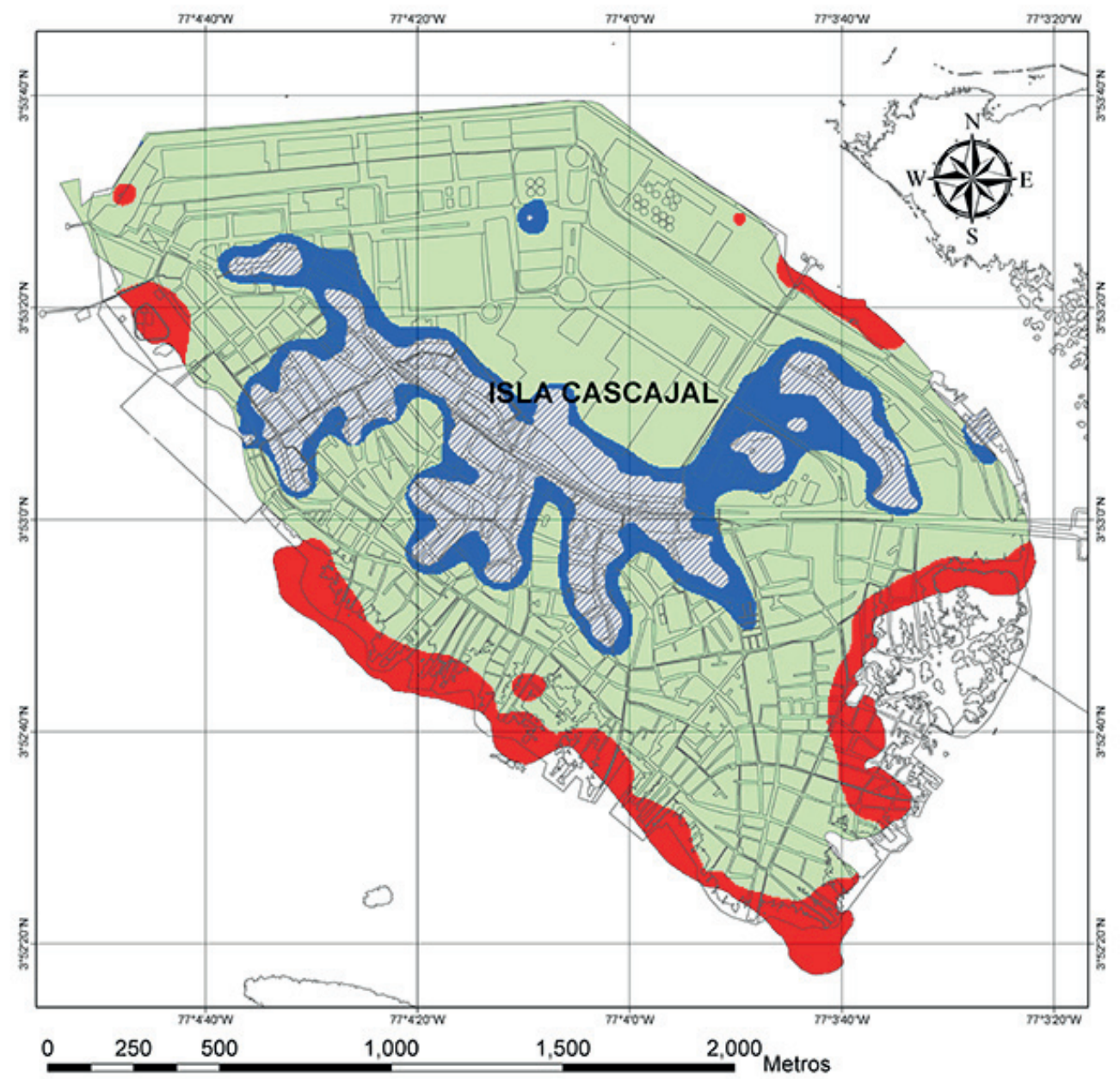

\begin{tabular}{|c|c|}
\hline \multicolumn{2}{|c|}{$\begin{array}{l}\text { EVALUACION DEL RIESGO HUMANO } \\
\text { INTERVALOS DE EDAD POBLACIONAL } \\
\text { LIMITACIONES O DISCAPACIOADES }\end{array}$} \\
\hline \multicolumn{2}{|c|}{$\begin{array}{l}\text { EXPOSICION ANTE } \\
\text { EVENTO TSUNAMIGÉNICO } \\
\text { SUBS - F75 - } 8.8 \mathrm{Mw} \\
\text { ISLA DE CASCAJAL }\end{array}$} \\
\hline INFORMACIÓN DE & REFERENCIA \\
\hline $\begin{array}{l}\text { Sistema de Referencia } \\
\text { Elipsoide } \\
\text { Origen de la Zona } \\
\text { Lathud del origen } \\
\text { Meridiano Central }\end{array}$ & $\begin{array}{l}\text { Datum Magna } \\
\text { WGS84 } \\
\text { Oeste } \\
4^{*} 35^{\circ} 46.32^{-2} \mathrm{~N} \\
77^{*} 4^{\prime} 39.03^{\prime \prime} \mathrm{W}\end{array}$ \\
\hline
\end{tabular}
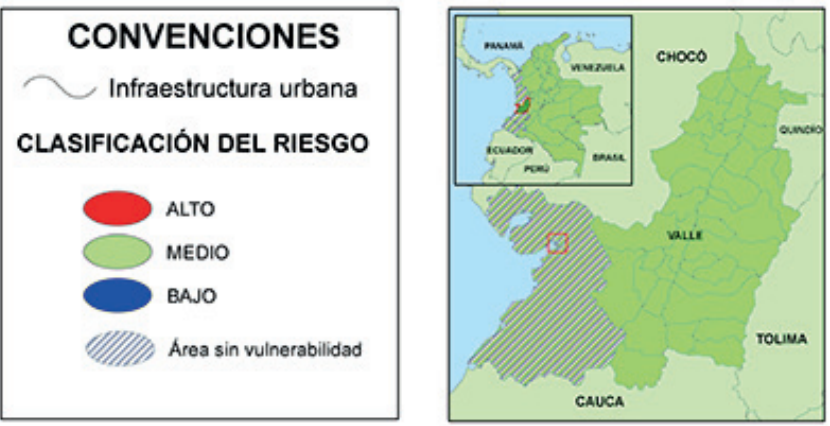

Fuente: Elaboración propia. 
Figura $N^{\circ} 18$

Riesgo de inundación a partir de la exposición estructural y económica

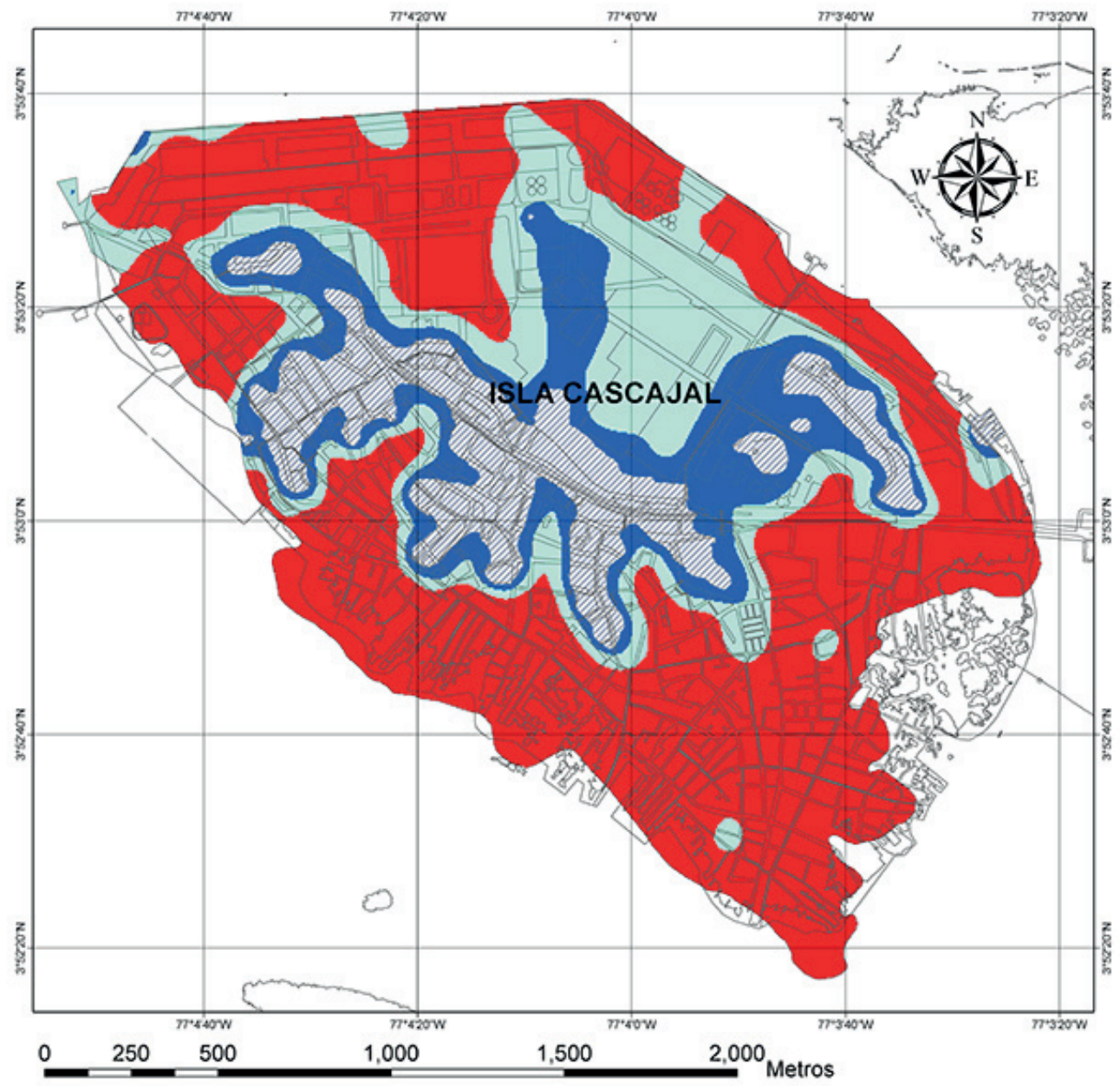

\begin{tabular}{|c|c|}
\hline \multicolumn{2}{|c|}{$\begin{array}{c}\text { EVALUACION DEL RIESGO } \\
\text { ELEMENTOS } \\
\text { ESTRUCTURALS } \\
\text { ECONOMICOS } \\
\text { TIPO DE VIVIENDA } \\
\text { COBERTURA SERVICIOS PÚBLICOS }\end{array}$} \\
\hline $\begin{array}{r}\text { EXPOSICION } \\
\text { EVENTO TSUNAM } \\
\text { SUBS-F75-8 } \\
\text { ISLA DE CASC }\end{array}$ & $\begin{array}{l}\text { ANIE } \\
\text { IGENICO } \\
8 \mathrm{Mw} \\
\text { AJAL }\end{array}$ \\
\hline INFORMACIÓN DE R & REFERENCIA \\
\hline $\begin{array}{l}\text { Sistema de Referencia } \\
\text { Elipsoide } \\
\text { Origen de la Zona } \\
\text { Lathud del origen } \\
\text { Meridiano Central }\end{array}$ & $\begin{array}{l}\text { Datum Magna } \\
\text { WGSB4 } \\
\text { Oeste } \\
4^{*} 35^{\circ} 46.32^{-} \mathrm{N} \\
77^{\prime} 4^{\prime} 39.03^{*} \mathrm{~W}\end{array}$ \\
\hline
\end{tabular}


Fuente: Elaboración propia. 


\section{Conclusiones}

Para el presente estudio, de acuerdo a los resultados obtenidos, se concluye que, para la Isla de Cascajal, el escenario de inundación por tsunami generado a partir de los eventos probabilísticos de amenaza crítica, presenta valores de inundación heterogéneos que afectarían de manera significativa la población e infraestructura urbana de dicha zona. Este evento sísmico de carácter tsunamigénico con una magnitud $8,8 \mathrm{Mw}$ en la fuente SUBS puede generar una altura máxima de inundación de 2,88 metros cubriendo un área de 3,32 km² que equivalen al 94,6"\% del área total de la Isla de Cascajal. Este escenario de amenaza crítica pone en riesgo directo (alto y medio) al $88,72 \%$ de la población vulnerable de la isla, mientras que las estructuras civiles con un piso de altura, presentan el mayor registro de riesgo alto $(83,4 \% \%)$.

Se recomienda establecer adquisición de información primaria en terreno, debido a que los datos utilizados tienen fecha de expedición del último censo nacional, para el año 2005, realizado por el Departamento Administrativo Nacional de Estadísticas (DANE), y para el cálculo de los parámetros de vulnerabilidad y riesgo no existen datos o aproximaciones acordes con el periodo de elaboración del presente estudio.

Por último, se resalta la importancia en el uso del software ejecutable CRISIS 2007 Ver 7.6 y los modelos geoestadísticos incorporados en el paquete de software ArcGIS, cuya aplicabilidad en el cálculo y comprensión de las variables y fenómenos sísmicos, permitieron establecer los escenarios y respectivos cálculos para determinación de los diferentes niveles de afectación para los elementos expuestos en el área de estudio.

\section{Referencias bibliográficas}

ABE, K. Quantification of tsunamigenic earthquakes by the $M_{t}$ scale. Tectonophysics, 1989, Vol. 166, p. $27-34$.

AGUILAR, A. CRISIS 99.1. Sistema de cómputo para el cálculo del peligro sísmico. México: Tesis de maestría, Universidad Nacional Autónoma de México (UNAM), 2001.

BRUNE, J.N. Tectonic Stress and the Spectra of Seismic Shear Waves from Earthquakes. Journal of Geophysical Research, 1970, Vol. 75, № 26, p. 4997 - 5009.

BRYANT, E. Tsunami: The underrate hazard. Cham: Springer International Publishing, $3^{\circ}$ edición, 2014.

CABALLERO, L. y ORTIZ, M. Evaluación del impacto de tsunamis en el litoral pacífico colombiano. Parte II (Región de Buenaventura). Boletín Científico CCCP, 2002, № 9, p. 45 - 57.

CARDONA, Y.; TORO, F.; VÉLEZ, J. y OTERO, L. Estimación de la amenaza por inundación generada por ondas de tsunami considerando la altura y velocidad de la lámina de agua inundante para el municipio de Tumaco. Boletín Científico CCCP, 2007, Vol. 14, p. 19 - 30. 
CHOI, B.H.; PELINOVSKY, E.; KIM, K.O. \& LEE, J.S. Simulation of the trans - oceanics tsunami propagation due to the 1883 Krakatau volcanic eruption. Natural Hazards and Earth System Sciences, 2003, Vol. 3, p. 321 - 332.

COCUÑAME, D.A. Modelamiento de escenarios de inundación por tsunami local para la Isla de Cascajal y los sectores turísticos de Juanchaco, Ladrilleros y la Barra. Cali: Tesis de pregrado, Departamento de Geografía, Universidad del Valle (UNIVALLE), 2016.

COLLOT, J.Y.; MARCAILLOU, B.; SAGE, F.; MICHAUD, F.; AGUDELO, W.; CHARVIS, P.; GRAINDORGE, D.; GUTSCHER, M.A. \& SPENCE, G. Are rupture zone limits of great subduction earthquakes controlled by upper plate structures? Evidence from multichanel seismic reflection data acquired across the northen Ecuador - southwest Colombia margin. Journal of Geophysical Research, 2004, Vol. 109, B11103, doi:10.1029/2004JB003060.

EVALUACIÓN DE RIESGOS NATURALES - AMÉRICA LATINA (ERN - Latina). Comprehensive Approach for Probabilistic Risk Assessment. 2011. Disponible en internet: http://ares.tu.chibau.jp/peru/pdf/ meeting/110309/CAPRA-English-Summary-2011.pdf

DENGLER, L.; GOLTZ, J.; FENTON, J.; MILLER, K. \& WILSON, R. Building tsunami - resilient communities in the United States: An example from California. Washington State Department of Natural Resources Tsulnfo Alert, 2011, Vol. 13, p. 1 - 12.

DEPARTAMENTO ADMINISTRATIVO NACIONAL DE ESTADíSTICAS (DANE). Boletín Censo General Perfil Buenaventura. 2005a. Disponible en internet:

http://www.dane.gov.co/files/censo2005/PERFIL_PDF_CG2005/76109T7T000.PDF

DEPARTAMENTO ADMINISTRATIVO NACIONAL DE ESTADÍSTICAS (DANE). Sistema de Consulta de Información Censal - Censo DANE 2005. 2005b. Disponible en internet: http://systema59. dane.gov.co/cgibin/RpWebEngine.exe/PortalAction?\&MODE=MAIN\&BASE=CG2005BASICO\&MAIN=WebServerMain.inl

DEPARTAMENTO ADMINISTRATIVO NACIONAL DE ESTADÍSTICAS. (DANE).). Geoportal DANE. Marco Geoestadístico Nacional por departamentos. 2005c. Disponible en Internet: https://geoportal. dane.gov.co/v2/?page=elementoDescargaMGN

DIRECCIÓN GENERAL MARÍTIMA - CENTRO DE INVESTIGACIONES OCEANOGRÁFICAS E HIDROGRÁFICAS DEL PACÍFICO (DIMAR - CCCP). Estudio de la Amenaza por Tsunami y Gestión del Riesgo en el Litoral Pacífico Colombiano. Bogotá: Editorial Dimar, Serie Publicaciones Especiales, 2013, Vol. 8, p. 18.

FOURNIER D'ALBE, E.M. Objetives of volcanic monitoring and monitoring and prediction. Journal of Geological Society, 1979, Vol. 136, p. $321-326$. 
GEIST, E.L. Source characteristics of the July 17, 1998, Papua New Guinea tsunami. EOS, Transactions of the American Geophysical Union, 1998, Vol. 79, p. 571.

GEIST, E.L. Local Tsunamis and Earthquake Source Parameters. Advances in Geophysics, 1999, Vol. 39, p. $117-209$.

GOTO, C.; OGAWA, Y.; SHUTO, N. \& IMAMURA, F. IUGG/IOC TIME Project: Numerical Method of Tsunami Simulation with the Leap - Frog Scheme. In: Intergovernmental Oceanographic Commission of UNESCO, IOC Manual and Guides, 1997, Vol. 35. Disponible en internet: http://unesdoc. unesco.org/images/0012/001223/122367eb.pdf

GREZIO, A., GASPARINI, P., MARZOCCHI, W., PATERA, A., and TINTI, S. Tsunami risk assessments in Messina, Sicily - Italy. Natural Hazards and Earth System Sciences, 2012, Vol. 12, p. $151-163$.

HERD, D.; YOUD, L.; MEYER, H.; ARANGO, J.; PERSON, W. \& MENDOZA, C. The Great Tumaco Colombia Earthquake of 12 December 1979. Science, 1981, Vol. 211, № 4481, p. $441-445$.

INSTITUTO COLOMBIANO DE GEOLOGÍA Y MINERÍA - DEPARTAMENTO ADMINISTRATIVO DE GESTIÓN DEL MEDIO AMBIENTE (INGEOMINAS - DAGMA). Estudio de microzonificación sísmica de Cali. Convenio No 02 de 2002. En: Subproyecto Evaluación de la Amenaza Sísmica Regional en Santiago de Cali. Cali: Instituto Colombiano de Geología y Minería, Informe 1-9, 2005.

KANAMORI, H. Mechanism of Tsunami Earthquakes. Physics of the Earth and Planetary Interiors, 1972, Vol. 6, p. 346-359.

KANAMORI, H. The energy release in great earthquakes. Journal of Geophysical Research, 1977, Vol. 82, p. $2981-2876$.

KANAMORI, H. \& MCNALLY, K.C. Variable rupture mode of the subduction zone along the Ecuador-Colombia coast. Bulletin of the Seismological Society of America, 1982, Vol. 72, p. 1241 - 1253.

KELLEHER, J. Rupture zone of large South American earthquakes and some predictions. Journal of Geophysical Research, 1972, Vol. 77, p. 2087 - 2103.

MARTÍNEZ, C.; ROJAS, O.; ARÁNGUIZ, R.; BELMONTE, A.; ALTAMIRANO, A. y FLORES, P. Riesgo de tsunami en caleta Tubul, Región del Biobío: Escenarios extremos y transformaciones territoriales posterremoto. Revista de Geografía Norte Grande, 2012, № 53, p. 85 - 106.

MARTÍNEZ, C. y ARÁNGUIZ, R. Riesgo de tsunami y planificación resiliente de la costa chilena. La localidad de Boca Sur, San Pedro de la Paz ( $\left.37^{\circ} \mathrm{S}\right)$. Revista de Geografía Norte Grande, 2016, Nº 64, Pp. $33-54$.

MOSQUERA, C. y PEÑA, C.M. Reevaluación de la amenaza sísmica en la ciudad de Cali por medio de un método de análisis integral de la información sismológica, geofísica y geológica. Cali: Tesis de pregrado, Escuela de Ingeniería Civil y Geomática, Universidad del Valle (UNIVALLE), 2010. 
NATIONAL EARTHQUAKE INFORMATION CENTER. Catálogo de eventos sísmicos destacados por subducción en el Pacífico colombiano, para el periodo de 1906 - 2015. Disponible en internet: http://earthquake.usgs.gov/contactus/golden/neic.php

OKAL, E.A. Seismic parameters controlling far - field tsunami amplitudes: A review. Natural Hazards, 1988, Vol. 1, p. $67-96$.

OKAL, E.A. \& SYNOLAKIS, C.E. Source discriminants for near - field. Geophysical Journal International, 2004, Vol. 158, p. $899-912$.

ORDAZ, M. Sismicidad y riesgo sísmico. Congreso Nacional de Ingeniería Sísmica, 1997, Vol. 1, p. $143-163$.

ORDAZ, M.; AGUILAR, A. \& ARBOLEDA, J. CRISIS 2007. Program for computing seismic hazard. Vers. 1.1. México: Instituto de Ingeniería, Universidad Nacional Autónoma de México (UNAM), 2007.

PAPOULIA, J.; TSAMBAS, A.; ANAGNOSTOU, C.; MAKRIS, L.; POMONIS, A.; GULKAN, P.; YALCINER, A.; SLEJKO, D.; MASCLE, J.; PAPADOPOULOS, G. \& NICOLICH, R. Seismic and Tsunami Risk Assessment and Mitigation Scenarios in the Western Hellenic Arc: The SEAHELLARC Project. In: 14th European Conference on Earthquake Engineering (ECEE), 2010, p. 8.

PARARAS - CARAYANNIS, C. Survey of the Earthquake and Tsunami of December 12, 1979, in CoIombia. International Tsunami Information Center Report, 1980, Vol. 13. №1, p.1-9.

POAG, C.W.; KOEBERL, C. \& REIMOLD, W.U. The Chesapeake Bay Impact Crater - Geology and Geophysics of a Late Eocene Submarine Impact Structure. Berlin: Springer - Verlag, 2004.

RAMÍREZ, J.E. y GOBERNA, J.R. Terremotos colombianos noviembre 23 y diciembre 12 de 1979. Informe Preliminar. Bogotá: Publicación del Instituto Geofísico de los Andes Colombianos, Universidad Javeriana. Serie A: Sismología, 1980, № 45.

RED SISMOLÓGICA NACIONAL DE COLOMBIA. Catálogo de sismicidad destacada en el Pacífico colombiano, para el periodo de 1993 - 2015. Disponible en internet: http://200.119.88.135/RSNC/ index.php/consultas

RESTREPO, J.C. y OTERO, L.J. Modelación numérica de eventos tsunamigénicos en la Cuenca Pacífica Colombiana - Bahía de Buenaventura. Revista Academia Colombiana de Ciencias, 2007, Vol. $31, N^{\circ} 120$, p. $363-377$.

RUDOLPH, E. \& SZIRTES, S. Das Kolumbianische Erdbeben am 31 januar 1906. Gerlands Beitrage zur geophysik, 1911, Vol. 11, № 1.Disponible en internet:

http://www.osso.org.co/docu/especiales/Traduccion_TERREMOTO_1906.pdf

RYBICKI, K. Dislocations and their geophysical applications. In: TIESSEYRE, R. (editor). Continuum Theories in Solid Earth Physics, Amsterdam: Elsevier, 1986, p. 18-186. 
SIEBERT, L. (editor). Landslides resulting from structural failure of volcanoes, Catastrophic Landslides: Effects, occurrence and mechanisms. Boulder: Geological Society of American, Reviews in Engineering Geology, 2004, Vol. 15.

STRUCTURAL ENGINEERS ASSOCIATION OF CALIFORNIA (SEAOC). Vision 2000 Report on performance based seismic engineering of buildings. Sacramento: Structural Engineers Association of California, 1995.

TINTI, S.; TONINI, R.; BRESSAN, A.; ARMIGLIATO, A.; GARDI, A.; GUILLANDE, R.; VALENCIA, N. \& SCHEER, S. Handbook of Tsunami Hazard and Damage Scenarios. SCHEMA (Scenarios for Hazard - induced Emergencies Management), Project N 030963. Joint Research Centre Scientific and Technical Reports, 2011, p. 1 - 41.

WARME, J.E. \& KUEHNER, H.C. Anatomy of an anomaly: the Devonian catastrophic Alamo impact breccia of southern Nevada. International Geology Review, 1998, Vol. 40, p. 189-2016.

WIECZOREK, G.F.; GEIST, E.L.; JAKOB, M.; ZIRNHELD, S.L.; BOYCE, E.; MOTYKA, R.J. \& BURNS, P. Landslide-induced wave hazard assessment - Tidal Inlet, Glacier Bay National Park, Alaska. 2004. Disponible en internet: https://pubs.usgs.gov/of/2003/ofr-03-100/ofr-03-100.html

ZITELLINI, N.; CHIERICI, F.; SARTORI, R.; \& TORELLI, L. The tectonic source of the 1755 Lisbon earthquake and tsunami. Annali Di Geofisica, 1999, Vol. 42, p. $41-55$. 
